\title{
The Asymptotic Distribution of Exponential Sums, I
}

\author{
S. J. Patterson
}

\section{CONTENTS}

1. Introduction

2. The Structure of Exponential Sums

3. Asymptotics of Arithmetic Functions

4. Computations and Results

5. Final Remarks

Appendix: The Numerical Evidence

References
2000 AMS Subject Classification: Primary 11L05;

Secondary 11N37, 11Y35

Keywords: Complete exponential sums, Gauss sums, arithmetic functions, Linnik-Selberg conjecture
Let $f(x)$ be a polynomial with integral coefficients and let, for $c>0, S(f(x), c)=\sum_{j(\bmod c)} \exp \left(2 \pi \imath \frac{f(j)}{c}\right)$. It has been possible, for a long time, to estimate these sums efficiently. On the other hand, when the degree of $f(x)$ is greater than 2 very little is known about their asymptotic distribution, even though their history goes back to C. F. Gauss and E. E. Kummer. The purpose of this paper is to present both experimental and theoretic evidence for a very regular asymptotic behaviour of $S(f(x), c)$.

\section{INTRODUCTION}

The purpose of this series of papers is to study the asymptotic distribution of exponential sums of the following types:

1. $S(f(x) ; c)=\sum_{\xi(\bmod c)} e(f(\xi) / c)$ where $e(u)=e^{2 \pi i u}$ and $f$ is a polynomial with integral coefficients,

$$
\begin{aligned}
& \text { 2. } K(a, b ; c)=\sum_{\substack{\xi(\bmod c) \\
\xi \bar{\xi} \equiv 1(\bmod c)}} e((a \xi+b \bar{\xi}) / c), \\
& \text { 3. } K_{\varepsilon}(a, b ; c)=\sum_{\xi \bar{\xi} \equiv 1(\bmod c)}\left(\frac{\xi}{c}\right) e((a \xi+b \bar{\xi}) / c) .
\end{aligned}
$$

The sums $K(a, b ; c)$ are classical Kloosterman sums and $K_{\varepsilon}(a, b ; c)$ are their twisted analogues, and are just about as classical as the $K(a, b ; c)$ themselves.

The reason for considering these together is that there are relationships between these different classes of sums - see, for example, [Duke and Iwaniec 93] and [Mao 97]. The estimation of individual sums of these types is well understood - see, for example, [Loxton and Vaughan 85], where a survey of known results is given. The purpose of the present investigation is to study the behaviour of these functions as functions of $c$. By and large most of the investigations in the past have concentrated on the case where $c$ is restricted to be a prime. In many cases, it appears that one obtains a much more complete picture

(c) A K Peters, Ltd. $1058-6458 / 2003 \$ 0.50$ per page Experimental Mathematics 12:2, page 135 
by considering general arguments $c$. Indeed, in the few cases of this type where it has been possible to obtain results, one begins by understanding the distribution over general values of $c$ and then uses a sieve method to analyze the case of primes - see [Heath-Brown 00], [HeathBrown and Patterson 79], and [Duke et al. 95] for examples of this kind.

The main motivation for investigating sums of this kind lies in diophantine analysis. The "circle method" and other techniques often rely on some form of Fourier analysis which "reduces" the problem in hand to one involving such sums. If we let $t(c)$ denote one of the sums above, then one would typically be interested in estimating sums of the type $\sum t(c) \omega(c)$ where $\omega$ is some reasonably well understood analytic or arithmetic function. For most applications, an estimate for the individual $t(c)$ suffices. However, better estimates are sometimes needed; for example, in [Linnik 63], Linnik studied a problem connected with quadratic forms and was led to propose the conjecture $\sum_{c \leq X} K(a, b ; c) / \sqrt{c}=\mathcal{O}\left(X^{\frac{1}{2}+\varepsilon}\right)$ for every $\varepsilon>0$. This conjecture is still unproven, but $\mathrm{N}$. V. Kuznetsov [Kuznetsov 80] has proved that it is true for $\varepsilon>\frac{1}{6}$. It is also worth noting, parenthetically, that $K(0,1 ; c)=\mu(c)$, the Möbius function, and so the conjecture $\sum_{c \leq X} K(0,1 ; c) / \sqrt{c}=\mathcal{O}\left(X^{\varepsilon}\right)$ for every $\varepsilon>0$ is equivalent to the Riemann hypothesis. Selberg [Selberg 65, page 9] indicated that a uniform version of Linnik's conjecture would imply the Ramanujan-Petersson conjecture; that was demonstrated by Ram Murty in [Murty 85].

Another motivation has come through the cohomological interpretation of exponential sums and other arithmetical functions. It is known that $K(a, b ; p)$ can be written as $2 \sqrt{p} \cos \phi_{p}(a, b)$ with $\phi_{p}(a, b) \in[0, \pi]$ whenever $p$ is a prime not dividing $a b$. By analogy with the theory of elliptic curves, it is believed that the $\phi_{p}(a, b)$ are distributed on $[0, \pi]$ over a large range of $p$ according to the Sato-Tate distribution-see [Serre 68, pages I-25, I-26]. Certain analogues of this have been provedsee [Adolphson 89] and [Katz 88] - but the framework of these results is rather distant from that described here.

In this paper, we shall analyze the problems and give a brief synopsis of the major conclusions drawn. More details will be given in later papers in this series.

\section{THE STRUCTURE OF EXPONENTIAL SUMS}

In this section, we shall summarize those aspects of the sums under consideration which we shall need. We begin with the two classes of Kloosterman sums $K$ and $K_{\varepsilon}$. If $c=c_{1} c_{2}$ with $c_{1}$ and $c_{2}$ co-prime, then we define $d_{1}, d_{2}$ so that $d_{1} c_{1} \equiv 1\left(\bmod c_{2}\right)$ and $d_{2} c_{2} \equiv 1\left(\bmod c_{1}\right)$. Then we have

$$
K(a, b ; c)=K\left(d_{2} a, d_{2} b ; c_{1}\right) K\left(d_{1} a, d_{1} b ; c_{2}\right)
$$

and

$$
K_{\varepsilon}\left(a, b ; c_{1} c_{2}\right)=K_{\varepsilon}\left(d_{2} a, d_{2} b ; c_{1}\right) \cdot K_{\varepsilon}\left(d_{1} a, d_{1} b ; c_{2}\right) .
$$

This reduces some problems to ones involving prime powers. If $k=1$, the results are due to Davenport, Hasse, and Weil; if $k>1$, they are due to Salié and are much easier. What we need is summarized in [Patterson 97]. We have, if $k \geq 1, p \nmid a b$

$$
K\left(a, b ; p^{k}\right)=\alpha\left(a, b ; p^{k}\right)+\alpha^{\prime}\left(a, b ; p^{k}\right),
$$

where

$$
\begin{gathered}
\alpha\left(a, b ; p^{k}\right) \cdot \alpha^{\prime}\left(a, b ; p^{k}\right)=p^{k} \\
\left|\alpha\left(a, b, p^{k}\right)\right|=\left|\alpha^{\prime}\left(a, b ; p^{k}\right)\right|=p^{k / 2} .
\end{gathered}
$$

In the case of $K_{\varepsilon}$ with $p \neq 2$, then we have an analogous statement.

If $p \mid a b$, then we have a number of different cases. We shall not record them all here, but refer to [Patterson 97, Prop. 2.1 and Th. 3.1], where details are given. We do note, however, that if $p^{k} \mid a$ and $p^{k} \mid b$, then

$$
K\left(a, b ; p^{k}\right)=(p-1) p^{k-1}
$$

which is of the form above with $\alpha_{p}(a, b)=p^{k}, \alpha_{p}^{\prime}(a, b)=$ $-p^{k-1}$, but now $\alpha_{p}(a, b) \cdot \alpha_{p}^{\prime}(a, b)=-p^{2 k-1}$. If $p$ $a, p \nmid b$, then $K(a, b ; p)=-1$ and so we have a different structure - as if the multiset $\left\{\alpha_{p}(a, b), \alpha_{p}^{\prime}(a, b)\right\}$ had been replaced by a singlet. If $k>1, p \nmid a, p \mid b$, then $K\left(a, b ; p^{k}\right)=0$ and $K_{\varepsilon}\left(a, b ; p^{k}\right)=0$.

It follows from this that if $c$ is co-prime to $a b$, then

$$
K(a, b ; c)=\sum_{\alpha \in A(a, b ; c)} \alpha \text { and } K_{\varepsilon}(a, b ; c)=\sum_{\alpha \in A_{\varepsilon}(a, b ; c)} \alpha
$$

where $|\alpha|=c^{\frac{1}{2}}$ in both cases and $A(a, b ; c)$ and $A_{\varepsilon}(a, b ; c)$ are multisets with $\operatorname{Card}(A(a, b ; c))=2^{\omega(c)}$, $\operatorname{Card}\left(A_{\varepsilon}(a, b ; c)\right)=2^{\omega(c)}$ where $\omega(c)$ is the number of prime divisors of $c$. In this case, one can define

$$
K^{(r)}(a, b ; c)=\sum_{\alpha \in A(a, b ; c)} \alpha^{r} \text { and } K_{\varepsilon}^{(r)}(a, b ; c)=\sum_{\alpha \in A_{\varepsilon}(a, b ; c)} \alpha^{r}
$$

where $r \geq 1$. From the point of view of understanding the Sato-Tate conjecture, these sums would be the most 
natural to study. Unfortunately, from both the theoretical and computational points of view, they are very difficult to work with. It seems, therefore, that at least at the present time, it is better to restrict one's attention to the $K(a, b ; c)^{r}$ and $K_{\varepsilon}(a, b ; c)^{r}$ if one wishes to study the value-distribution of these Kloosterman sums. They are at least exponential sums in their own right and restricted to primes contain the same information as the $K^{(r)}(a, b ; c)$ and $K_{\varepsilon}^{(r)}(a, b ; c)$. In a series of papers [Fouvry and Michel 02, Fouvry and Michel 03a, Fouvry and Michel 03b], E. Fouvry and P. Michel have proved some very interesting results about the value distribution of the values of Kloosterman and similar sums. They combine large sieve methods with the structure theory of these sums obtained from the algebraic geometric interpretation.

The $S(f ; c)$ are rather more subtle. It is convenient to introduce some concepts from [Loxton and Vaughan 85]. If $f$ is a polynomial with integral coefficients and degree $n$, we let $\xi_{1}, \ldots, \xi_{m}$ be the roots with multiplicities $e_{1}, \ldots, e_{m}$. Let $K$ be the subfield of $\mathbb{C}$ generated by the $\xi_{1}, \ldots, \xi_{m}$ and $\mathcal{D}(f)$ be the different of $f$, i.e., the fractional ideal generated by the $\frac{f^{\left(e_{i}\right)}\left(\xi_{1}\right)}{e_{i} !}$; in [Loxton and Smith 82], it is remarked that this is an integral ideal in $K$. For $c \in \mathbb{N}$, let $(c, \mathcal{D}(f))$ be $\prod_{p \mid c} p^{\min \left(\operatorname{ord}_{p}(\mathcal{D}(f)), \operatorname{ord}_{p}(c)\right)}$ where for an ideal $\mathfrak{a}$ of $K$, we define

$$
\operatorname{ord}_{p}(\mathfrak{a})=\max \left\{k / l: p^{k} \mid \mathfrak{a}^{l}, k, l>0\right\} .
$$

Let $e(f)=\max _{1 \leq i \leq m} e_{i}$; this is called the exponent of $f$. Let

$$
C_{n}=\prod_{\substack{p \leq n \\ p \text { prime }}} p
$$

Then ([Loxton and Vaughan 85, Theorem 2]) one has

$$
|S(f ; c)| \leq C_{n}^{\frac{1}{e+1}} \cdot(n-1)^{\omega(c)}\left(\mathcal{D}\left(f^{\prime}\right), c\right)^{\frac{1}{e+1}} c^{1-\frac{1}{e+1}}
$$

where $e=e\left(f^{\prime}\right)$. This suggests that $S\left(f, p^{k}\right)$ behaves as if it were the sum of $n-1$ terms of modulus $p^{k\left(1-\frac{1}{e+1}\right)}$, but, in fact, the structure is usually much more complicated. We give two examples which will be relevant later. For the value distribution of such sums, see also [Fouvry and Michel 03a, Michel 98].

First of all, we have for $p \nmid n, p \nmid a$ :

$$
\begin{aligned}
S\left(a x^{n} ; p^{k}\right) & =\sum_{\substack{\chi^{n}=1 \\
\chi \neq 1}} g(\chi, a, p) & & (k=1) \\
& =p^{k-1} & & (2 \leq k \leq n) \\
& =p^{n-1} S\left(a x^{n} ; p^{k-n}\right) & & (k>n) .
\end{aligned}
$$

In this case, the exponent of $f^{\prime}$ is $n-1$. Here, $g(\chi, a, p)$ is the Gauss sum

$$
\sum_{x} \chi(x) e\left(\frac{a x}{p}\right) ;
$$

note that each of these summands has modulus $p^{\frac{1}{2}}$. In particular, in this example, $S\left(a x^{n} ; p\right)$ will be zero if $(n, p-1)=1$. This example, having, as it does, the maximal exponent possible, represents an extreme case. These evaluations are classical-see, for example, [Vaughan 81, $\S 4.2]$.

The other case which we shall consider is rather more special, namely $f(x)=x^{4}+a x^{2}$. Here, $e\left(f^{\prime}\right)=1$ when $a \neq 0$. By [Weil 48], one has $S\left(x^{4}+a x^{2} ; p\right)=\alpha+\alpha^{\prime}+\alpha^{\prime \prime}$ where $|\alpha|=\left|\alpha^{\prime}\right|=\left|\alpha^{\prime \prime}\right|=p^{\frac{1}{2}}$. In fact, one can be rather more specific. We can write $S\left(x^{4}+a x^{2}, p\right)$ as $\sum_{y(p)}\left(1+\left(\frac{y}{p}\right)\right) e\left(\frac{y^{2}+a y}{p}\right)$. This splits naturally into two sums; the first is a standard quadratic Gauss sum which is $\sqrt{p} \cdot \gamma_{p} \cdot e\left(\frac{\overline{4} \cdot a^{2}}{p}\right)$ where

$$
\begin{aligned}
& \gamma_{p}=1 \quad p \equiv 1 \quad(\bmod 4) \\
& =i \quad p \equiv 3 \quad(\bmod 4)
\end{aligned}
$$

and $\overline{4} \cdot 4 \equiv 1(\bmod p)$. Note that $\overline{4}=\frac{p+1}{4}$ if $p \equiv 3$ $(\bmod 4)$ and $\overline{4}=-\frac{p-1}{4}$ if $p \equiv 1(\bmod 4)$, so that this sum is, for $p$ large,

$$
\begin{array}{ll}
\sqrt{p}\left(1+\mathcal{O}\left(\frac{1}{p}\right)\right) & \text { if } p \equiv 1(\bmod 4) \text { and } a \text { is even, } \\
-\sqrt{p} i\left(1+\mathcal{O}\left(\frac{1}{p}\right)\right) & \text { if } p \equiv 1(\bmod 4) \text { and } a \text { is odd, } \\
\sqrt{p} i\left(1+\mathcal{O}\left(\frac{1}{p}\right)\right) & \text { if } p \equiv 3(\bmod 4) \text { and } a \text { is even, } \\
-\sqrt{p}\left(1+\mathcal{O}\left(\frac{1}{p}\right)\right) & \text { if } p \equiv 3(\bmod 4) \text { and } a \text { is odd. }
\end{array}
$$

By [Weil 48], $\sum_{y(p)}\left(\frac{y}{p}\right) e\left(\frac{y^{2}+a y}{p}\right)$ is of the form $\alpha^{\prime}+\alpha^{\prime \prime}$ with $\left|\alpha^{\prime}\right|=\left|\alpha^{\prime \prime}\right|=p^{\frac{1}{2}}$. In the case of $k>1$, we have a similar phenomenon, now easily proved by the "method of stationary phase" (see [Livné and Patterson 02, Proposition $5.2])$. We find

$$
\begin{aligned}
S\left(x^{4}+a x^{2} ; p^{k}\right)= & p^{\frac{k}{2}} \cdot\left(1+\left(1+\left(\frac{2 a}{p}\right)\right) e\left(\frac{3 \cdot \overline{4} \cdot a^{2}}{p^{k}}\right)\right) \\
& \text { if } k \text { is even. } \\
= & p^{\frac{k}{2}} \gamma_{p}\left(1+\left(1+\left(\frac{2 a}{p}\right)\right) e\left(\frac{3 \cdot \overline{4} a^{2}}{p^{k}}\right)\right)
\end{aligned}
$$$$
\text { if } k \text { is odd } k>1 \text {. }
$$

The arguments used here can be adapted to the case of polynomials of the form $f_{0}\left(x^{2}\right)$.

We shall now return to the "generic case," i.e., when $e\left(f^{\prime}\right)=1$. For $k=1$, Weil's theorem yields the decomposition $S(f(x) ; p)=\alpha_{1}+\ldots+\alpha_{n-1}$. For $k>1$, the 
"method of stationary phase" yields

$$
S\left(f(x) ; p^{k}\right)=\left\{\begin{array}{cc}
p^{\frac{k}{2}} \sum_{\xi: f^{\prime}(\xi) \equiv 0} e\left(\frac{f(\xi)}{p^{k}}\right) & \\
& \text { if } \left.k \text { is even } p^{k}\right) \\
p^{\frac{k}{2}} \cdot \gamma_{p} \sum_{\xi: f^{\prime}(\xi) \equiv 0}\left(\bmod p^{k}\right) & e\left(\frac{f(\xi)}{p^{k}}\right) \\
& \text { if } k \text { is odd. }
\end{array}\right.
$$

It is easy to see that the number of summands can vary between 0 and $n-1$; this means that attempting a general definition of $S^{(k)}(f ; c)$ is futile. The number of factors is described by the zeta-function of the splitting field of $f^{\prime}(x)$.

It is worth noting here that for $a \in \mathbb{Z}$,

$$
\begin{aligned}
S(f+a ; c) & =S(f ; c) e\left(\frac{a}{c}\right) \\
& =S(f ; c)\left(1+\mathcal{O}\left(\frac{1}{c}\right)\right) \quad \text { as } c \rightarrow \infty .
\end{aligned}
$$

Thus, we can alter $f$ by addition of a constant without altering the asymptotic behaviour of $S(f ; c)$. Likewise, we can replace $f(x)$ by $f(x+c)$ or $f(-x+c)$ with $c \in \mathbb{Z}$ without altering the asymptotic behaviour of $S(f(x), c)$.

Finally, we shall recall results in three cases which are relevant to us. The first is based on the reciprocity formula for quadratic Gauss sums for $A, c$ positive

$$
\begin{aligned}
\sum_{x} e\left(\frac{A x^{2}+B x}{c}\right) & =\frac{1+i}{2} \\
\cdot \frac{c^{\frac{1}{2}}}{A^{\frac{1}{2}}} & \sum_{y} e\left(-\frac{c y^{2}+2 B y}{4 A}\right) e\left(\frac{-B^{2}}{4 A c}\right)
\end{aligned}
$$

see, for example, [Berndt, et al. 98, Theorem 1.2.2]; to obtain the result in the form quoted here, one sets the parameters $a, b$, and $c$ of that theorem to the values $a=$ $2 A, b=2 B, c=c$ for which $a c+b$ is clearly even. This formula gives a "closed expression" for $S\left(A x^{2}+B x ; c\right)$ which we can use directly. Although this case is very special, it is very helpful for orientation. For us, the major consequence is the following theorem:

Theorem 2.1. Let $A>0$; let $R$ be the largest number $r$ with $r^{2} \mid A$.

$\sum_{c \leq X} S\left(A x^{2}+B x ; c\right)=\left\{\begin{array}{cc}\frac{2(1+i)}{3} R A^{-\frac{1}{2}} X^{\frac{3}{2}}+\mathcal{O}\left(X^{\frac{1}{2}}\right) \\ \mathcal{O}\left(X^{\frac{1}{2}}\right) & \text { if } B \equiv 0 \quad(\bmod R) \\ & \text { otherwise. }\end{array}\right.$

Proof: We use the reciprocity law and consider $c$ restricted to an individual residue class $(\bmod 4 A)$, say $c \equiv \alpha(\bmod 4 A)$. Since we have for any natural number $N$ the elementary estimate

$$
\sum_{\substack{c \leq X \\ c \equiv \alpha}} c^{\frac{1}{2}}=\frac{2}{3} N^{-1} X^{\frac{3}{2}}+\mathcal{O}\left(x^{\frac{1}{2}}\right)
$$

and as we can absorb $e\left(\frac{-B^{2}}{4 A c}\right)=1+\mathcal{O}\left(c^{-1}\right)$ into the error term, we obtain

$$
\begin{aligned}
\sum_{c \leq X} S\left(A x^{2}+B x\right. & ; c)=\frac{1+i}{3} A^{-\frac{1}{2}}(4 A)^{-1} \\
& \cdot \sum_{\alpha, y} e\left(-\frac{\alpha y^{2}+2 B y}{4 A}\right) X^{\frac{3}{2}}+\mathcal{O}\left(X^{\frac{1}{2}}\right) .
\end{aligned}
$$

Now the sum of $e\left(-\frac{\alpha y^{2}}{4 A}\right)$ over $\alpha$ is a geometric series which becomes $4 A$ if $y^{2} \equiv 0(\bmod 4 A)$ and zero otherwise. This yields

$$
\begin{aligned}
\sum_{c \leq X} S\left(A x^{2}+B x ; c\right)= & \frac{1+i}{3} A^{-\frac{1}{2}} \\
& \cdot \sum_{y} e\left(-\frac{2 B y}{4 A}\right) X^{\frac{3}{2}}+\mathcal{O}\left(X^{\frac{1}{2}}\right),
\end{aligned}
$$

where the sum over $y$ is taken over those residue classes $(\bmod 4 A)$ for which $y^{2} \equiv 0(\bmod 4 A)$. The condition that $y^{2} \equiv 0(\bmod 4 A)$ means that $y \equiv 0$ $(\bmod (4 A) /(2 R))$ as follows from the prime decomposition. If we write $y=(2 A / R) \eta$ where $\eta$ is taken $(\bmod 2 R)$. The sum over $\eta$ is easy to carry out and we obtain the result stated above.

We note here that the coefficient of the real and imaginary parts of the coefficient are positive or zero. In this case, they are also equal. We could have considered the asymptotics over certain arithmetic sequences by essentially the same methods. In some more general cases, the details can be rather involved, but at least if we consider the asymptotic behaviour over a sequence $c \equiv 0$ $(\bmod D)$ for a fixed $D$.

The second example is the main theorem of [Patterson 02]. It states

Theorem 2.2. For $A \in \mathbb{Z}, A \neq 0$, there is a constant $k(A)$ so that, for $\varepsilon>0$,

$$
\sum_{c \leq X} S\left(A x^{3}, c\right)=k(A) X^{\frac{4}{3}}+\mathcal{O}\left(X^{\frac{5}{4}+\varepsilon}\right)
$$

with

$$
k(A)=\frac{(2 \pi)^{2 / 3} \Gamma\left(\frac{1}{3}\right)}{24 \cdot L\left(2,\left(\frac{-3}{\cdot}\right)\right)} \prod_{p^{k} \| A} T_{p}\left(p^{k}\right) \cdot A^{-\frac{1}{3}}
$$


where

$$
\begin{aligned}
& T_{p}\left(p^{k}\right)= p^{[k / 3]}+p^{[(k-1) / 3]}-p^{-1} \\
& \text { if } \quad k \geq 1, p \equiv 1 \quad(\bmod 3) \\
&=\left(p^{[k / 3]}+p^{[(k-1) / 3]}\right) \frac{1+p^{-1}}{1+p^{-2}}-\frac{2 p^{-1}}{1+p^{-2}} \\
& \text { if } \quad k \geq 1, p \equiv 2 \quad(\bmod 3) \\
&= 3^{\left[\frac{k}{3}\right]}+3^{\left[\frac{k-1}{3}\right]}-\frac{1}{3} \\
& \text { if } \quad k \geq 1, p=3 .
\end{aligned}
$$

One can also obtain rather more extensive results over $\mathbb{Z}[\omega]$ where $\omega$ is a primitive third root of 1 . Let us embed $\mathbb{Z}[\omega]$ in $\mathbb{C}$ by $\omega=\exp \left(\frac{2 \pi \imath}{3}\right)$. For $z \in \mathbb{Z}[\omega]$, let $e_{\omega}(z)=$ $\exp (2 \pi \imath(z+\bar{z}))$ and, for $A, B \in \sqrt{-3}^{-1} \mathbb{Z}[\omega]$ and $c \in \mathbb{Z}[\omega]$, let

$$
S_{\omega}\left(A x^{3}+B x ; c\right) \sum_{j} e_{\omega}\left(\frac{A j^{3}+B j}{c}\right)
$$

where the summation is in $\mathbb{Z}[\omega]$. R. Livné and the author proved in [Livné and Patterson 02, Theorems 1.2, 1.3] the results summarized in the following theorem.

Theorem 2.3. Suppose that $A, B, D, D^{\prime} \in \mathbb{Z}[\omega]$ are such that $D, D^{\prime}$ are square-free and mutually co-prime, that both are co-prime to 3 , that any prime $(\neq \lambda)$ which divides $B$ also divides $D^{\prime}$, that $27 . A \mid B^{3}$, and that $B^{3} /(27 . A) \equiv \pm 1(\bmod 3)$. Let $\chi$ be a Dirichlet character of modulus dividing $D^{\prime}$. Then for any $\varepsilon>0$, we have

$$
\begin{gathered}
\sum_{\substack{\mathrm{N}(c) \leq X,\left(c, D^{\prime}\right)=(1) \\
(\bmod D), c \equiv 1 \\
(\bmod 3)}} S_{\omega}(A, B ; c) \chi(c)= \\
\frac{(2 \pi)^{1 / 3} \Gamma(1 / 3)^{2} \sqrt{3}}{32 \zeta_{\mathbb{Q}[\omega]}(2)} \frac{\mathrm{N}\left(D^{\prime}\right)}{\sigma\left(D D^{\prime}\right)} X^{4 / 3}+\mathcal{O}\left(X^{5 / 4+\varepsilon}\right) .
\end{gathered}
$$

if $\chi=\left(\frac{A}{.}\right)_{3}$ and

$$
\sum_{\substack{\mathrm{N}(c) \leq X,\left(c, D^{\prime}\right)=(1) \\(\bmod D), c \equiv 1}} S_{\omega}(A, B ; c) \chi(c)=\mathcal{O}\left(X^{5 / 4+\varepsilon}\right)
$$

otherwise.

Suppose next that $D, D^{\prime} \in \mathbb{Z}[\omega], D, D^{\prime} \equiv 1(\bmod 3)$ are mutually co-prime and square-free. Suppose that the only primes dividing $A \in \mathbb{Z}[\omega]$ are those dividing $3 D^{\prime}$. Suppose $\chi$ is a Dirichlet character to the modulus $D^{\prime}$. Then if $\chi^{3}$ is not principal, we have

$$
\sum_{\substack{\mathrm{N}(c) \leq X, c \equiv 0 \\ c \equiv 1}(\bmod 3),\left(c, D^{\prime}\right)=1} S_{\omega}(A, 0 ; c) \chi(c) \cdot(1-\mathrm{N}(c) / X)=\mathcal{O}\left(X^{\frac{5}{4}+\varepsilon}\right)
$$

If $\chi=\left(\frac{\dot{\sigma}}{\delta_{1} \delta_{2}^{2}}\right)_{3}$ with $\delta_{1} \delta_{2} \mid D^{\prime}, \delta_{1}, \delta_{2} \equiv 1(\bmod 3)$, then there exist $C^{\prime}\left(A, D, D^{\prime}, \delta_{1}, \delta_{2}\right)$ and $C^{\prime \prime}\left(A, D, D^{\prime}, \delta_{1}, \delta_{2}\right)$ so that, for $\varepsilon>0$,

$$
\begin{gathered}
\sum_{\substack{\mathrm{N}(c) \leq X, c \equiv 0) \\
c \equiv 1}} S_{\omega}(A, 0 ; c) \chi(c) \cdot(1-\mathrm{N}(c) / X)= \\
\quad C^{\prime}\left(A, D, D, D^{\prime}\right)=1 \\
\quad+C^{\prime \prime}\left(A, D, \delta_{1}, \delta_{2}\right) X^{\frac{4}{3}} \log X \\
\\
\left.\quad \delta_{1}, \delta_{2}\right) X^{\frac{4}{3}}+\mathcal{O}\left(X^{\frac{5}{4}+\varepsilon}\right) .
\end{gathered}
$$

Moreover, if $C^{\prime}\left(A, D, D^{\prime}, \delta_{1}, \delta_{2}\right)=0$, then

$$
C^{\prime \prime}\left(A, D, D^{\prime}, \delta_{1}, \delta_{2}\right)=0 \text {. }
$$

Unfortunately, in [Livné and Patterson 02, Theorem 1.2], the condition above, "that any prime $(\neq \lambda)$ which divides $B$ also divides $D^{\prime}$," the phrase " $(\neq \lambda)$ " was omitted, rendering the theorem empty. More details about the value of $C^{\prime}\left(A, D, D^{\prime}, \delta_{1}, \delta_{2}\right)$ can be found in [Livné and Patterson 02, Theorem 1.3].

These results summarize about all that is known about the global behaviour of exponential sums of Hua type. A few minor, but insubstantial, extensions are possible. It is worth noting that all of the results have certain restrictions. These are most painful in the last theorem where one would very much like to ease the restrictions on the coefficients $A, B$. Note also that in the behaviour in the cases $B=0$, there is a dichotomy between Theorem 2.1 and Theorem 2.3 in the case $B=0$ that is reminiscent of that between the divisor function $d(n)$ and the function $r(n)$ giving the number of representations of $n$ as a sum of two squares.

\section{ASYMPTOTICS OF ARITHMETIC FUNCTIONS}

Let $t$ be an arithmetic function of the type that we have introduced above. The basic type of asymptotic behaviour which would be of interest to us is whether $\sum_{c \leq X} t(c)$ behaves like $K \cdot X^{\gamma}$ as $X \rightarrow \infty$. One would like to extend this to arithmetic progressions. Since there are numerous linear relations between sums over different arithmetic progressions, it is convenient to have a method free of redundancy. To achieve, this let $\chi$ be a primitive Dirichlet character and $D \in \mathbb{N}$. Then we ask whether

$$
\sum_{c \equiv 0} t(c) \chi(c / D)
$$

has an asymptotic behaviour of the form $K(\chi, D) X^{\gamma(\chi, D)}$. Let $A$ be an arithmetic progression. It is then not difficult to express $\sum_{c \leq X} t(c)$ in terms

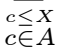


of the $\sum_{\substack{c \leq X \\ c \equiv 0}} t(c) \chi(c / D) ;$ see [Patterson $\left.D\right)$ details.

For applications - for example, to the analysis of the distribution of the function $t$ over primes - one might expect that these suffice, but unfortunately, this is not so. In the case of the Möbius function, one would have

$$
\sum_{c \equiv 0} \mu(c) \chi(c / D)=O\left(X^{\frac{1}{2}+\varepsilon}\right)
$$

for all $\varepsilon>0$ if the generalized Riemann Hypothesis holds. However, $\sum_{c \leq X} \mu(c) \Lambda(c) \sim-X$. One might hope that the general sieve identity

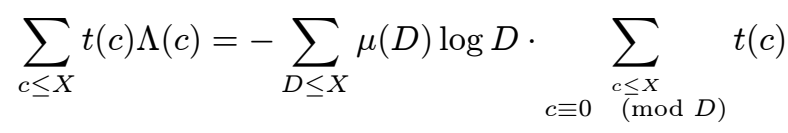

would indicate the behaviour of $\sum_{c \leq X} t(c) \Lambda(c)$, but this example shows that such hopes are naive. One is led to investigate also such sums as

$$
\sum_{c \equiv 0} \mu(c) t(c) \cdot \chi(c / D)
$$

or, more generally,

$$
\sum_{c \equiv 0} t(c) \cdot t^{*}(c) \chi(c / D),
$$

where $t^{*}$ is another arithmetic function.

As in considerations of this sort, the range of potential functions can easily become much too large to be manageable so we have restricted ourselves to the following cases for the moment:

- the $S(f(x), c)$ for $f(x)=A x^{3}+B x$ over $\mathbb{Z}$,

- more general $S(f(x), c)$ of degree 3 and also some polynomials $f(x)$ of degree 4 and 5 ,

- the behaviour of $K(a, b ; c) \mu(c)$ and $K_{\epsilon}(a, b ; c) \mu(c)$,

- the behaviour of $K(a, b ; c) K\left(a^{\prime}, b^{\prime} ; c\right)$ and $K_{\epsilon}(a, b ; c) K_{\epsilon}\left(a^{\prime}, b^{\prime} ; c\right)$.

We have not investigated $K(a, b ; c)$ itself, as the Linnik-Selberg conjecture predicts its behaviour and Kuznetsov's theorem, assuming Selberg's conjecture on the eigenvalues of the Laplace-Beltrami operator, supports this, [Kuznetsov 80]; for results toward the Selberg conjecture, see [Luo, Rudnick and Sarnak 95] and for more on the distribution of Kloosterman sums, see [Fouvry and Michel 03b]. Likewise, we have not investigated $K_{\varepsilon}(a, b ; c)$ as the analogue of Kuznetsov's method for forms of $\frac{1}{2}$-integral weight should lead to a result of the form

$$
\sum_{\substack{c \leq X \\(\bmod D)}} K_{\varepsilon}(a, b ; c) \chi(c / D) \sim k(a, b ; \chi ; D) X^{\frac{5}{4}}
$$

with an explicit formula for $k(a, b ; \chi ; D)$.

Up to the present, this does not seem to have been treated in the literature - see, however, [Duke et al. 95] and the reference to the $\mathrm{PhD}$ thesis of I. Vardi on page 245 of [Goldfeld and Sarnak 83]; the results of this thesis have apparently not been published. In [Duke et al. 95], Duke, Friedlander, and Iwaniec prove the analogue of Hooley's theorem [Hooley 64] on the equidistribution of solutions of quadratic congruences over prime moduli. To do this, they use a version of Kuznetsov's theorem for forms of half-integral weight. For their purposes, they can avoid a detailed analysis of the sort that would be necessary to determine the $k(a, b ; \chi ; D)$ above explicitly.

Naturally, all of these could be considered as more general exponential sums, but this opens up a field of investigation too wide to be so contemplated.

The expectation is then that for each sum $t(c)$, there will be an exponent $\gamma(t)$ and a coefficient $k_{t}(\chi, D)$ so that

$$
\sum_{c \equiv 0} t(c) \chi(c / D) \sim k_{t}(\chi, D) \cdot X^{\gamma(t)}
$$

for $X \rightarrow \infty$.

In this paper, we shall discuss the general answer to the second problem. In the next paper of this series, we shall discuss the finer details of the theory of the polynomials $A x^{3}+B x$ and in the third, both the behaviour of the Kloosterman sums and the distributions of the various arithmetic functions.

\section{COMPUTATIONS AND RESULTS}

The actual numerical experiments needed to investigate the

$$
\sum_{c \equiv 0} t(c) \chi(c / D)
$$

are relatively straightforward, but there are one or two points worth mentioning, as they affect the range available. Each $t(c)$ involves at least $\mathcal{O}(c)$ computations, sometimes $\mathcal{O}(c \log c)$ and so the entire computational requirements grow like $\mathcal{O}\left(X^{2}\right)$ or $\mathcal{O}\left(X^{2} \log X\right)$. It was 
found that using any dynamical method of representing numbers (such as the PARI/gp package) was too slow because of the "garbage collection." Consequently, the programs were written in $C$ and integers had a wordlength of 32 bits. This meant that there was a risk of overflow, which is often difficult to spot in this sort of computation. The safest, and fastest, way of computing the polynomials was by "Russian multiplication" and continually reducing $(\bmod c)$.

For most of the examples considered, one has that the general order of magnitude of $t(c)$ was $c^{\frac{1}{2}} \cdot 2^{\omega(c)}$. This means that one expects a "noise level" of $\mathcal{O}\left(X^{1+\varepsilon}\right)$ in

$$
\sum_{c \equiv 0} t(c) \chi(c / D),
$$

and this itself should have a dominant term $k_{t}(\chi, D) \cdot X^{\gamma}$. In the examples studied, $\gamma$ was generally $\frac{4}{3}$ or $\frac{5}{4}$ and $k_{t}(\chi, D)$ in general moderately small, usually less than 1. This would mean that to obtain an accuracy of, say, $k$ decimal places, one would need, on a conservative estimate, $\log _{10} X \geq \frac{k+1}{\gamma-1}$ computational steps. If $\gamma=\frac{4}{3}$ and $k=2$, we would then need $X \geq 10^{9}$. With $k=1$, one would need $X \geq 10^{6}$. Since the number of operations needed to compute this is a multiple of $X^{2}$, one sees that whereas the case $k=1$ is feasible, but expensive, the case $k=2$ is entirely impossible. Indeed, the amount of information obtained is proportional to $\log X$, and the cost to $X^{2}$. The best compromise appeared to be $X$ around 100000 or 500000 . In principle, this scarcely suffices for even one significant digit in $k_{t}(\chi, D)$, but one could at least get an idea. Since here $\log X<12$, the range was much too small to use any sort of "denoising" techniques. From one point of view, the use of Riesz means, i.e.,

$$
\sum_{c \equiv 0} \underset{c \leq X}{(\bmod D)}(c) \chi(c / D)\left(1-\frac{c}{X}\right)
$$

should be more stable and theoretically more plausible; it unfortunately gives disproportionately excessive weight to the small values of $c$ and this distorts the result. These considerations indicate that one has to treat all results here with a fair degree of skepticism. In particular, there is little possibility of identifying the $k_{t}(\chi, D)$ in terms of known constants.

The numerical experiments indicated the following conclusions:

Conjecture 4.1. If $f$ is of degree $n$, then

$$
\sum_{\substack{c \leq X \\ c \equiv 0}} S(f(x), c) \chi(c / D) \sim k(\chi, D) X^{\gamma}
$$

where $\gamma=1+\frac{1}{n}$ or $1+\frac{2}{n}$, the second case arising only if there exist $a \in \mathbb{Z}$ with $f(a-x)=f(x)$ and $\operatorname{deg}(f)>2$.

One has $\operatorname{Re}(k(1,1)) \geq 0$; if the leading coefficient is positive, then $\operatorname{Im}(k(1,1)) \geq 0$.

In these $\gamma$ and $k(\chi, D)$ are the parameters associated with $S(f ; c)$. The exponent in the generic case is the one suggested by the analysis of [Livné and Patterson 02]. Those cases where the exponent is expected to be $1+\frac{2}{n}$ can be regarded as a reflection of the description of $S\left(x^{4}+a x^{2} ; c\right)$ given in Section 2 and its generalizations. This is in no way a rigorous argument.

The second assertion can be understood as an asymptotic form of Gauss' theorem for higher Gauss sums. It should be noted that since replacing $f(x)$ by $f(-x)$ yields the same sums, it follows that

$$
\operatorname{Im}(k(1, D))=0
$$

if $\operatorname{deg}(f)$ is odd.

The computations up to the present are not sufficient to make hypotheses of type 2 above whenever $(\chi, D) \neq(1,1)$. It appears that many of the $k(\chi, D)$ can be zero, but, in view of the discussion above, it is hard to distinguish between "small" and "zero." The example of Theorem 2.1 shows that the coefficients can be zero without the summands being zero and the example below, of $3 x^{3}+2 x^{2}$, shows that the same apparently happens here.

The positivity posited in 2 indicates that one might expect that $\operatorname{Re}(k(\chi, D))$ and $\operatorname{Im}(k(\chi, D))$ are given as infinite products of the type of the "singular series" of the circle method. So far, no plausible hypothesis has been found. This is what happens in the case of Theorem 2.2 .

We shall give here some typical examples:

- $x^{3}+2 x$ with $\gamma=\frac{4}{3}$

- $2 x^{3}-x$ with $\gamma=\frac{4}{3}$

- $x^{3}+2 x^{2}$ with $\gamma=\frac{4}{3}$

- $3 x^{3}+x^{2}+x$ with $\gamma=\frac{4}{3}$

- $x^{4}$ with $\gamma=\frac{3}{2}$

- $x^{4}+x$ with $\gamma=\frac{5}{4}$

- $x^{4}+x^{2}$ with $\gamma=\frac{3}{2}$

- $2 x^{4}+4 x^{3}+6 x^{2}+4 x+1$ with $\gamma=\frac{3}{2}$

- $x^{4}+2 x^{3}+3 x^{2}+2 x+1$ with $\gamma=\frac{3}{2}$ 
Note that $2 x^{4}+4 x^{3}+6 x^{2}+4 x+1$ is equal to $x^{4}+(1+x)^{4}$ and $x^{4}+2 x^{3}+3 x^{2}+2 x+1$ is equal to $\frac{x^{4}+1+(1+x)^{4}}{2}$ and so both are invariant under $x \mapsto-1-x$.

In each of the cases above, we reproduce the graph of $X \mapsto X^{-\gamma} \sum_{c \leq X} S(f(x), c)$ in the range $X \leq 100,000$. The graphs are sampled at intervals of 100 except for the second case which is sampled at intervals of 500. Although there is considerable cancellation, the asymptotic behaviour is extremely marked. It is apparent that the sampling rate also is not very critical. The value of $\gamma$ chosen is based partly on general principles and on the numerical evidence. It should be remembered that small variations in the exponent are difficult to recognize numerically in a range of this limited logarithmic length.

The graphs are reproduced at the end of this paper. The fact that these represent the sort of asymptotic behaviour conjectured is clear. Indeed, considering the extensive cancellation that has to occur, the behaviour is much more pronounced than one would have dared to expect. Nevertheless, it is more or less impossible to guess the constants involved.

In the error term, there are two types that one normally meets. The first is that which occurs in Riemann's explicit formula where the error term is a sum of terms of the form $c(\rho) X^{\rho}$. This gives a fairly irregular behaviour because it reflects the fact that the generating Dirichlet series has poles, probably not too far to the right of the line representing the main term. Note that the sums are often, as in the case of Riemann's explicit formula, not absolutely convergent, but nevertheless they offer a good representation of the actual asymptotic behaviour. Another example of this type is the hyperbolic circle problem. In other cases, one has the asymptotic formulæ of Voronoi type, that is analogous to those in the circle or divisor problems. These are made up of terms (summed over $\lambda) c(\lambda, \mu) X^{\alpha} \cos \left(\lambda X^{\beta}+\mu\right)$ and this reflects generally cases where the asymptotic behaviour is limited by the growth in vertical strips of the generating Dirichlet series and not by the existence of poles. Two classical examples are the formulae

$$
\sum_{n \leq X} 1=X-\frac{1}{2}+\frac{1}{2 \pi} \sum_{m \geq 1} \frac{\sin (2 \pi m X)}{m}
$$

where $\alpha=0 \beta=1$ and $\mu=-\frac{\pi}{2}$ and the Voronoi formula itself

$$
\sum_{n \leq X} r(n)=\pi X-1+\sqrt{X} \sum_{m \geq 1} \frac{r(m)}{m^{\frac{1}{2}}} J_{1}(2 \pi \sqrt{m X}),
$$

where $r(n)$ is the number of representations of $n$ as a sum of two squares, $\alpha=\frac{1}{4}, \beta=\frac{1}{2}$, and $\mu=-\frac{3 \pi}{4}$. Both of these formulae are only conditionally convergent and have to be carefully interpreted at integer arguments $X$. In them, the terms $-\frac{1}{2}$ and -1 , respectively, reflect the existence of a pole and also a monotonic component in the behaviour of $X^{-1} \sum_{n \leq X} 1$ and $X^{-1} \sum_{n \leq X} r(n)$, respectively. The same type of behaviour seems to be visible in a number of the graphs presented here, but in view of the tenuity of the evidence, we shall not speculate further here. One should recall that this behaviour also occurs in formulae of Riemann type if one nondominant pole is further to the right than all others. More information on the structure of the "error term" would be a prerequisite to using the present information to obtain better estimates of the constants appearing in the asymptotic formula.

One should also note that the graphs are rougher the smaller $\gamma$ is. There are reasons to believe that the error term, even after smoothing, remains of order $\Omega\left(X^{1-\eta}\right)$ for any $\eta>0$ Thus, the smaller the value of $\gamma$, the smaller the signal-to-noise ratio is.

\section{FINAL REMARKS}

There are a number of remarks that one should make about the observation above. First of all, it is far from clear that the formulation above is the correct one. One beguiling alternative is the following. In [Weil 64, Chapter II] Weil reformulated Gauss' theorem in the form $\int_{k_{\mathbb{A}}} e_{\mathbb{A}}\left(x^{2}\right) d x=1$ where $k_{\mathbb{A}}$ denotes the adele ring and $e_{\mathbb{A}}$ is a nontrivial additive character on $k_{\mathbb{A}}$, trivial on $k$. This suggests that one should perhaps study, in place of the $S(f ; c)$, the integrals

$$
c \mapsto \int e_{\mathbb{A}}(f(x) / c) d x,
$$

where we keep $e_{\mathbb{A}}$ fixed. We shall examine the integral in the special case $f(x)=a x^{3}+b x(a, b \in \mathbb{Z}, k=\mathbb{Q})$. It is worth examining this integral. As $k=\mathbb{Q}$, we take $e_{\infty}(x)=e^{2 \pi i x}$.

Then, if

$$
A i(u)=\int_{-\infty}^{+\infty} e^{2 \pi i\left(x^{3}-u x\right)} d x
$$

denotes the Airy integral (see [Copson 65, Chapter 9] with a slight change of notation), we have if $a, c>0$

$$
\int_{\mathbb{Q}_{\infty}} e_{\infty}(f(x) / c) d x=\left(\frac{c}{a}\right)^{\frac{1}{3}} \cdot A i\left(\frac{-b}{a^{\frac{1}{3}} c^{\frac{2}{3}}}\right) .
$$

Moreover, by Nicolson's formula (see [Copson 65, page 99]) or [Watson 22, page 190], we have 


$$
\begin{aligned}
& A i(u)=\frac{2 \pi}{3}\left(\frac{u}{3}\right)^{\frac{1}{2}}\left\{J_{\frac{1}{3}}\left(\frac{2 \cdot 2 \pi}{3^{\frac{3}{2}}} u^{\frac{3}{2}}\right)+J_{-\frac{1}{3}}\left(\frac{2 \cdot 2 \pi}{3^{\frac{3}{2}}} u^{\frac{3}{2}}\right)\right\} \\
& \text { if } u>0 \\
& =\frac{(2 \pi)^{\frac{1}{3}}}{3} u^{\frac{1}{2}} K_{\frac{1}{3}}\left(\frac{2 \cdot 2 \pi}{3^{\frac{3}{2}}} u^{\frac{3}{2}}\right) \quad \text { if } u<0 \\
& A i(0)=\frac{1}{(2 \pi)^{\frac{1}{3}} \sqrt{3}} \Gamma\left(\frac{1}{3}\right) \text {. }
\end{aligned}
$$

It follows that for $c:|c|_{\infty} \rightarrow \infty$,

$$
\int_{\mathbb{Q}_{\infty}} e_{\infty}(f(x) / c) d x \sim\left(\frac{c}{a}\right)^{\frac{1}{3}} 3^{-\frac{1}{2}} \Gamma\left(\frac{1}{3}\right) .
$$

If $p \times 3 a c$, then it is easy to see that

$$
\int_{\mathbb{Q}_{p}} e(f(x) / c) d x=1
$$

if the Haar measure is so normalized that $\mathbb{Z}_{p}$ has measure 1. The remaining factors can be represented as a sum. We shall consider the case $b \neq 0$. It is then most convenient to rewrite the integral as

$$
\int e_{p}\left(\frac{a c^{2}}{b^{3}} x^{3}+x\right) d x \cdot\left|\frac{c}{b}\right|_{p} .
$$

Let $\sigma_{p}(u)=\int_{\mathbb{Q}_{p}} e_{p}\left(u \cdot x^{3}+x\right) d x$.

This can be computed by splitting the integral into subintegrals over $|x|_{p} \leq R$ and $|x|_{p}=R \cdot p^{l}(l \geq 1)$.

We can choose $R$ so that these latter integrals are all 0 and the first is an exponential sum. Let $t=\operatorname{ord}_{p}(u)$. Then we find

$$
\begin{aligned}
& \sigma_{p}(u)=p^{\frac{t}{3}} \quad t \leq 0, t \equiv 0 \quad(\bmod 3) \\
&=p^{\frac{t-1}{3}} \quad t \leq 0 ; t \equiv 1 \quad(\bmod 3) \\
&=p^{t-\frac{2}{3}} \sum_{\xi(p)} e_{p}\left(u \cdot p^{-t} \cdot \xi^{3} / p\right) \quad t \leq 0, t \equiv 2 \quad(\bmod 3) \\
&=\sum_{\xi\left(p^{\frac{t}{2}}\right)} e_{p}\left(\frac{u p^{-t} \xi^{3}+\xi}{p^{\frac{t}{2}}}\right) \quad t>0, t \equiv 0 \quad(\bmod 2) \\
&=\sum_{\xi\left(p^{\frac{t-1}{2}}\right)} e_{p}\left(\frac{u p^{-t} \cdot p \xi^{3}+\xi}{p^{\frac{t-1}{2}}}\right) \\
&=\sum_{\xi\left(p^{\frac{t+3}{2}}\right)} e_{p}\left(\frac{u p^{-t} \xi^{3}+p \xi}{p^{\frac{t+3}{2}}}\right) \quad(\bmod 2), p \neq 3 \\
& t>0, t \equiv 1 \quad(\bmod 2), p=3 .
\end{aligned}
$$

In point of fact, we do not need these evaluations. It comes out the same if we replace our original exponential sum by $\frac{1}{M^{2}} \sum_{x\left(c M^{3}\right)} e\left(\frac{a x^{3}+M^{2} b x}{M^{3} c}\right)$ where $M$ is "divisible enough"; this means that for each prime $p$, we should have

$$
\operatorname{ord}_{p}(M) \geq
$$

$\max \left(\frac{1}{3}\left(\operatorname{ord}_{p}(3 a)-1\right), \frac{1}{2}\left(\operatorname{ord}_{p}(3 a)-\operatorname{ord}_{p}(b)-1\right), 0\right)$.

The sum above can be interpreted as an integral and so does not depend on $M$. One can understand this as restricting the class of $f(x)=a x^{3}+b x$ and moduli $D$ considered above. One might suspect, although there is little evidence for it, that this class may be better behaved than the more general case.

There is one final point that needs to be addressed. In [Livné and Patterson 02, §8] and [Patterson 97], we examine the distribution of the sums

$$
S(a ; c)=\sum_{x(c)} e\left(\frac{a x^{3}}{c}\right)
$$

where now $c \in \mathbb{Z}[\omega], x$ is taken $(\bmod c)$ in $\mathbb{Z}[\omega]$ and $e(v)=e^{2 \pi i(v+\bar{u})}$. In those cases which can be treated, one has, for a certain $K(a) \in \mathbb{C}$,

$$
\sum_{c: N(c) \leq X} S(a, c) \sim K(a) \cdot X^{\frac{4}{3}} \cdot \log X .
$$

In one present case, we have conjectured that the logarithm factor does not appear. This deserves comment. We recall that over $\mathbb{Z}$ the sums $\sum_{x(c)} e\left(a x^{3} / c\right)$ vanish if $c$ contains a prime factor $p$ with $p \equiv 2(\bmod 3)$. Since $\mathbb{Q}(\omega)$ contains the third roots of 1 , nothing of this kind occurs in this case. The situation can be considered as analogous to the arithmetic functions $r(n)$ (the number of representations of $n$ as a sum of two squares) and $d(n)$ (the number of divisors of $n$ ). Then $r(n)=0$ if $n$ is divisible (exactly) by an odd power of a prime $p \equiv-1$ $(\bmod 4)$. Nothing of this kind happens with $d$. On the other hand, $\sum_{n \leq X} r(n) \sim \pi X$, but $\sum_{n \leq X} d(n) \sim X \log X$. This shows that $d$ is analogous to the exponential sum in the case of $\mathbb{Q}(\omega)$, and $r$ in the case of $\mathbb{Q}$.

The fact that we do not have a logarithmic factor is associated with vanishing of the sum on a very large set of arguments. If one is prepared to speculate on the analogues of the conjecture above in the case of an arbitrary number field (or $\mathbb{A}$-field), then it is necessary to treat the case of such polynomials separately which are invariant under a transformation of the form $f(\zeta x+b)=f(x)$ where $b$ is an integer of the field and $\zeta$ is a primitive $n^{\text {th }}$ root of 1 . The evidence above shows that when the field is $\mathbb{Q}$, the asymptotic behaviour in the case of quadratic $f$ has an exponent $\gamma=1+\frac{1}{2}$, but if $\operatorname{deg}(f)>2$ and such a condition is satisfied, then $\gamma=1+\frac{2}{n}$. A complete picture is not available at present. 
Experimental Mathematics, Vol. 12 (2003), No. 2

6. APPENDIX: THE NUMERICAL EVIDENCE

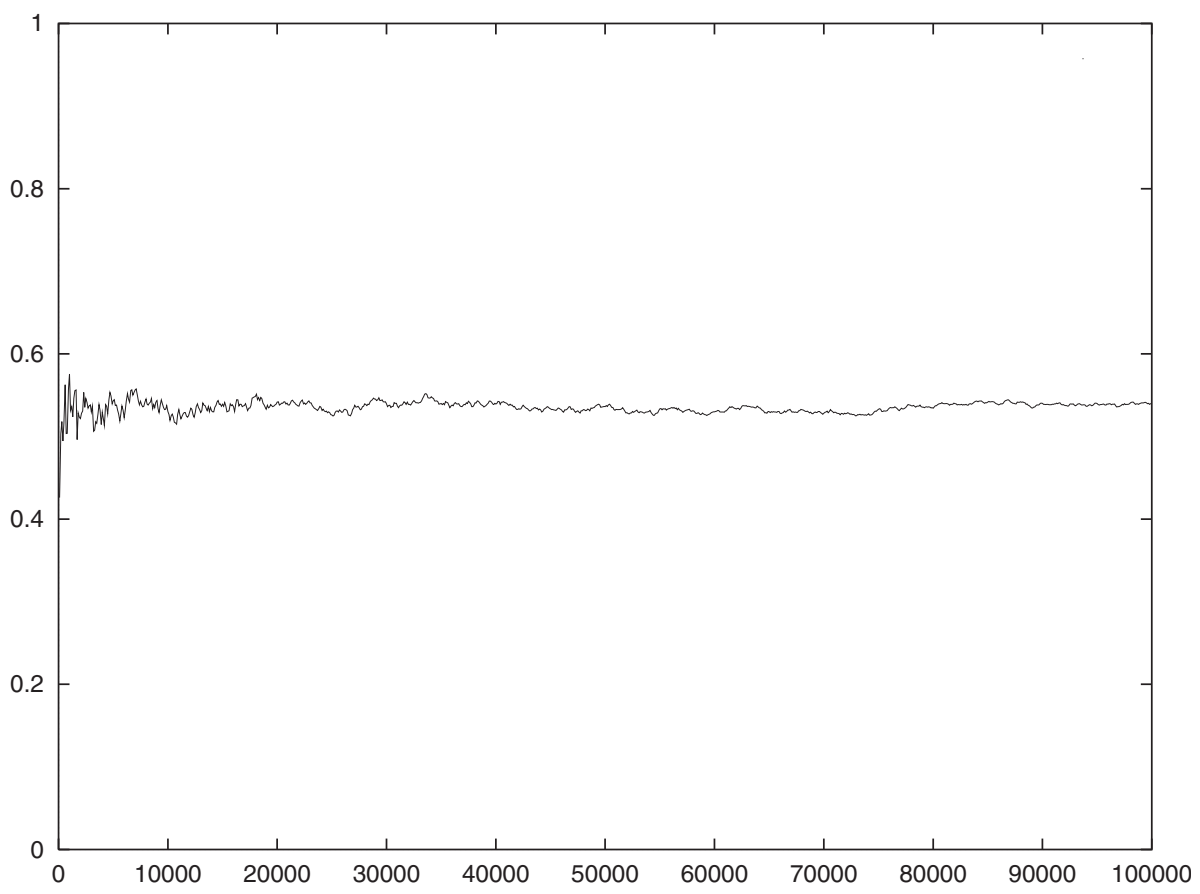

FIGURE 1. The asymptotic behaviour in the case of $x^{3}+2 x, \gamma=\frac{4}{3}$.

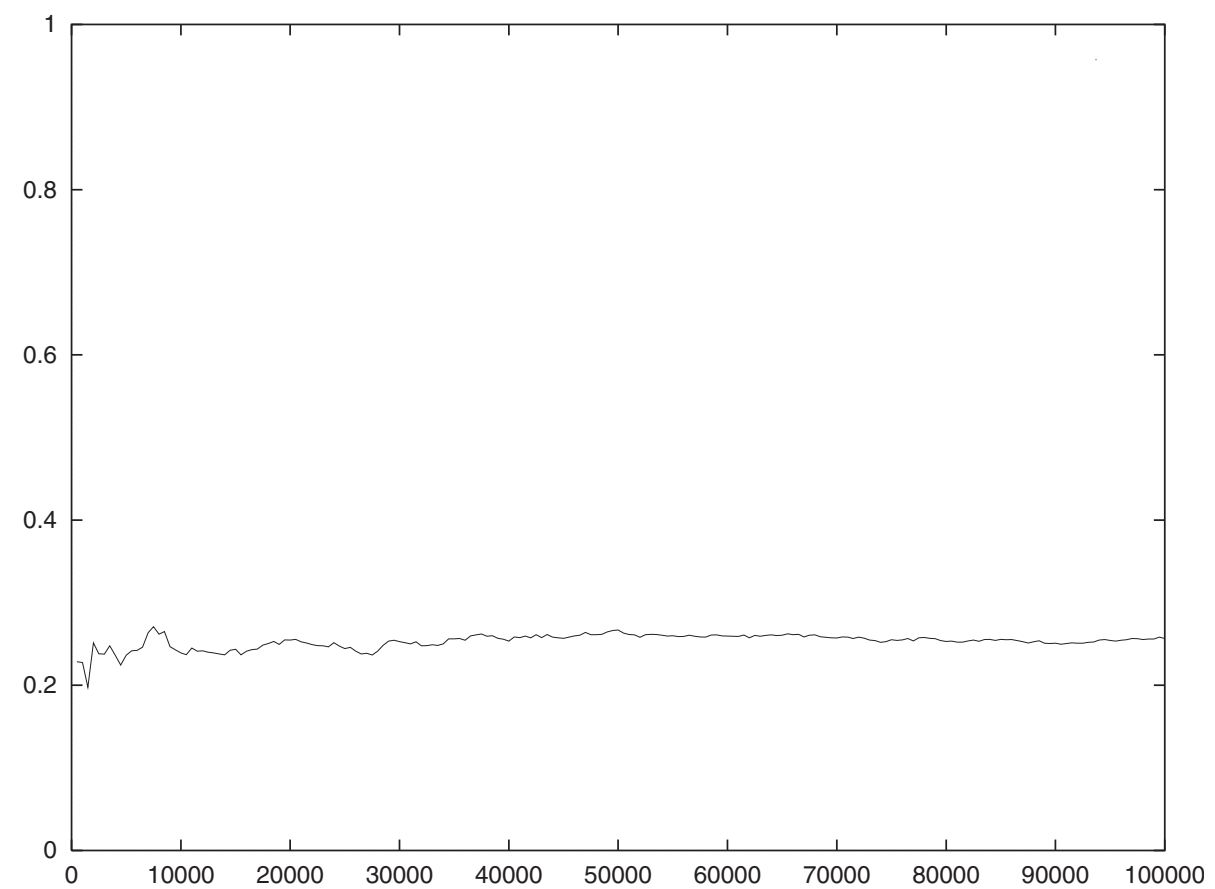

FIGURE 2. The asymptotic behaviour in the case of $2 x^{3}-x, \gamma=\frac{4}{3}$ 


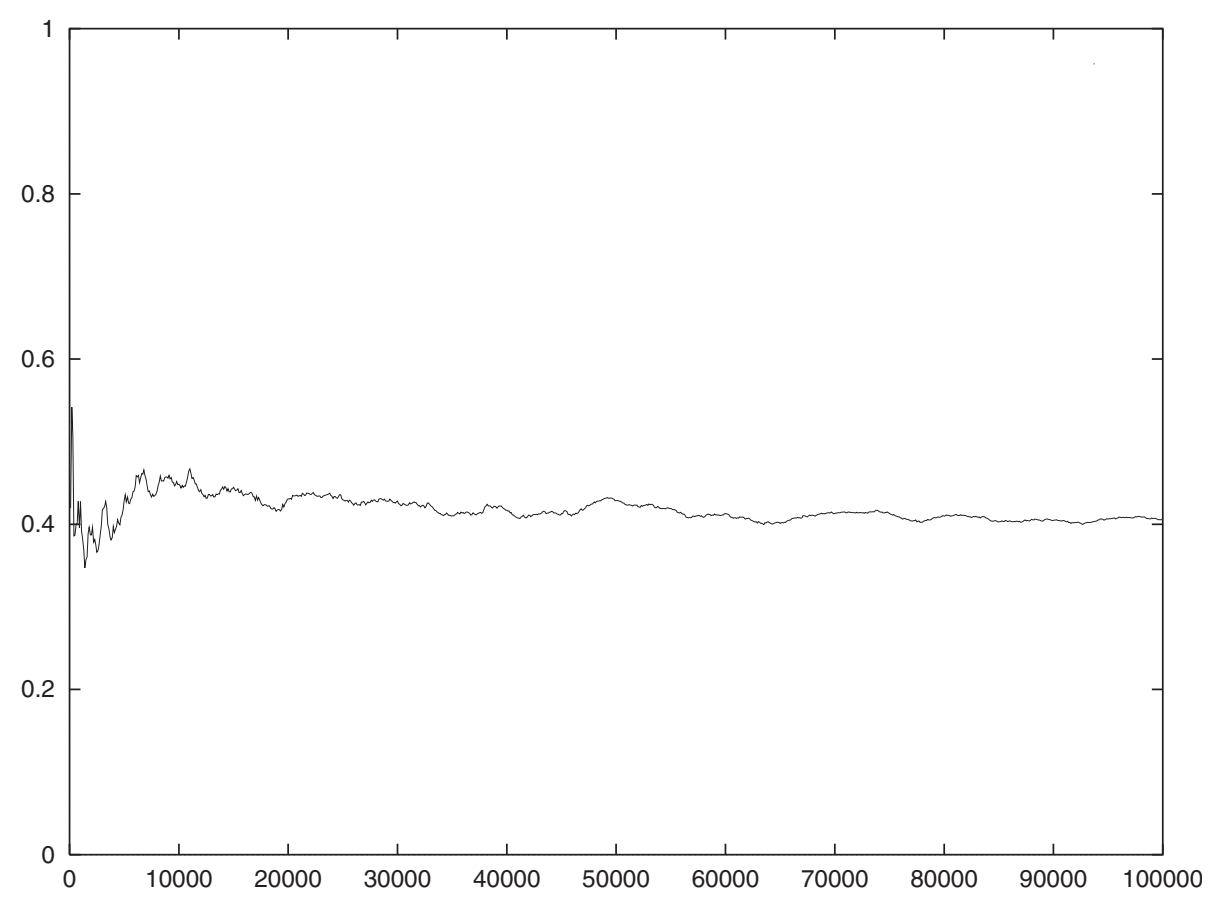

FIGURE 3. The asymptotic behaviour in the case of $3 x^{3}+2 x^{2}$ (real part), $\gamma=\frac{4}{3}$.

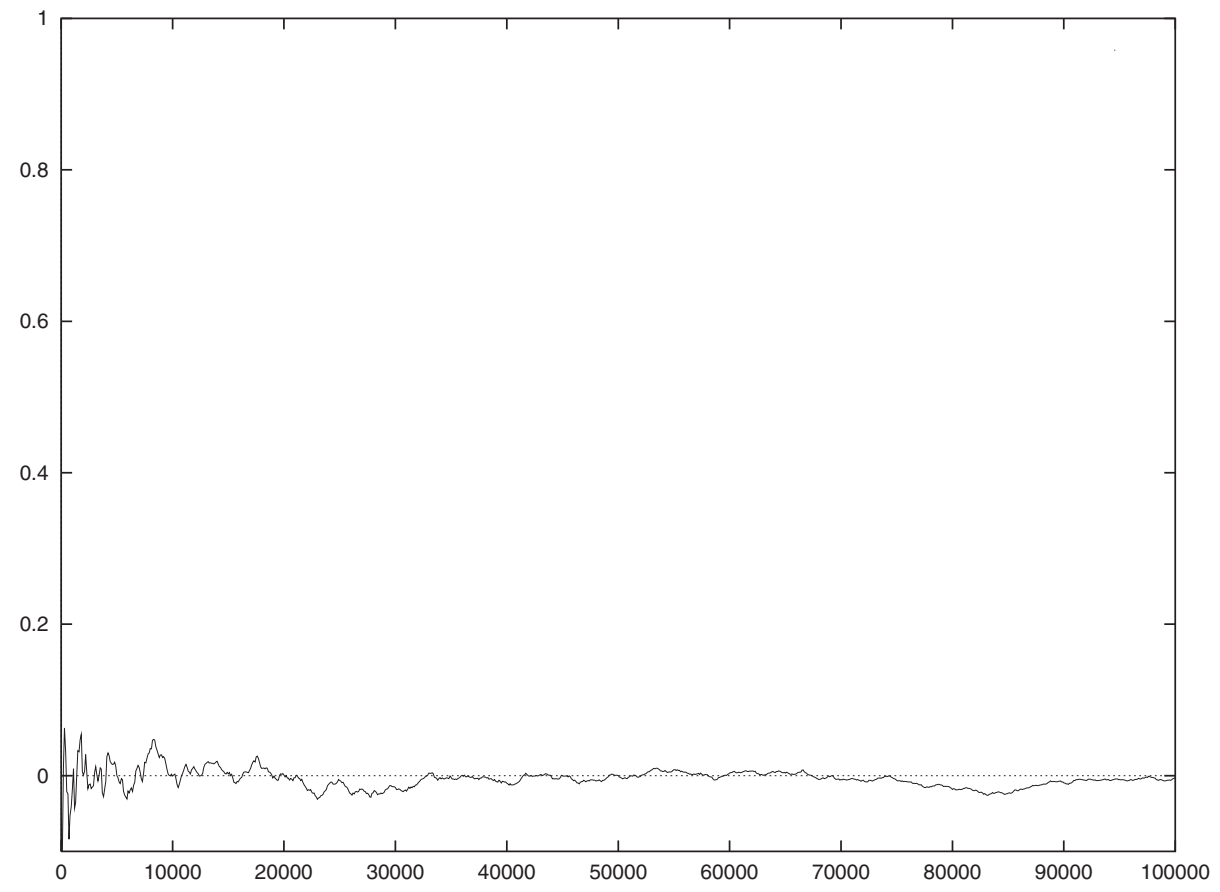

FIGURE 4. The asymptotic behaviour in the case of $3 x^{3}+2 x^{2}$ (imaginary part), $\gamma=\frac{4}{3}$. 


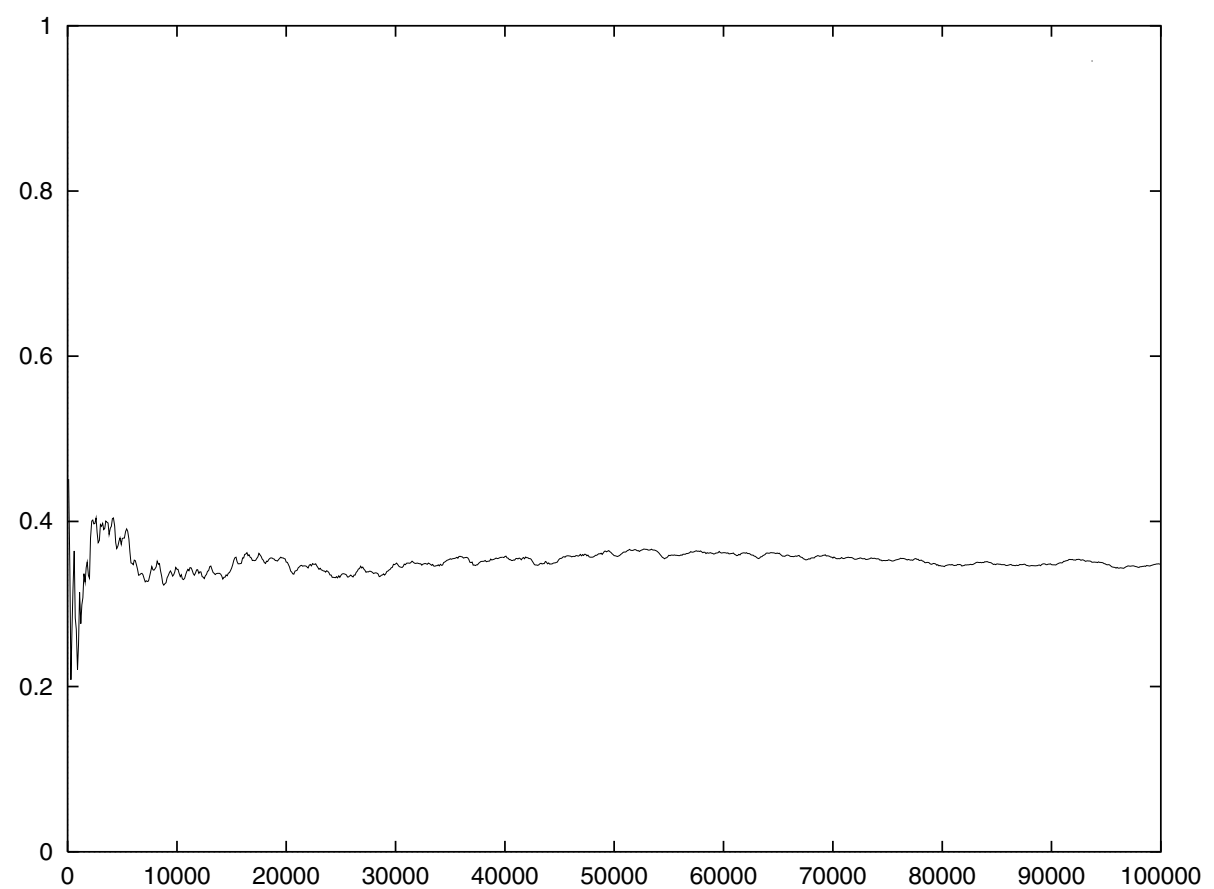

FIGURE 5. The asymptotic behaviour in the case of $3 x^{3}+x^{2}+x$ (real part), $\gamma=\frac{4}{3}$.

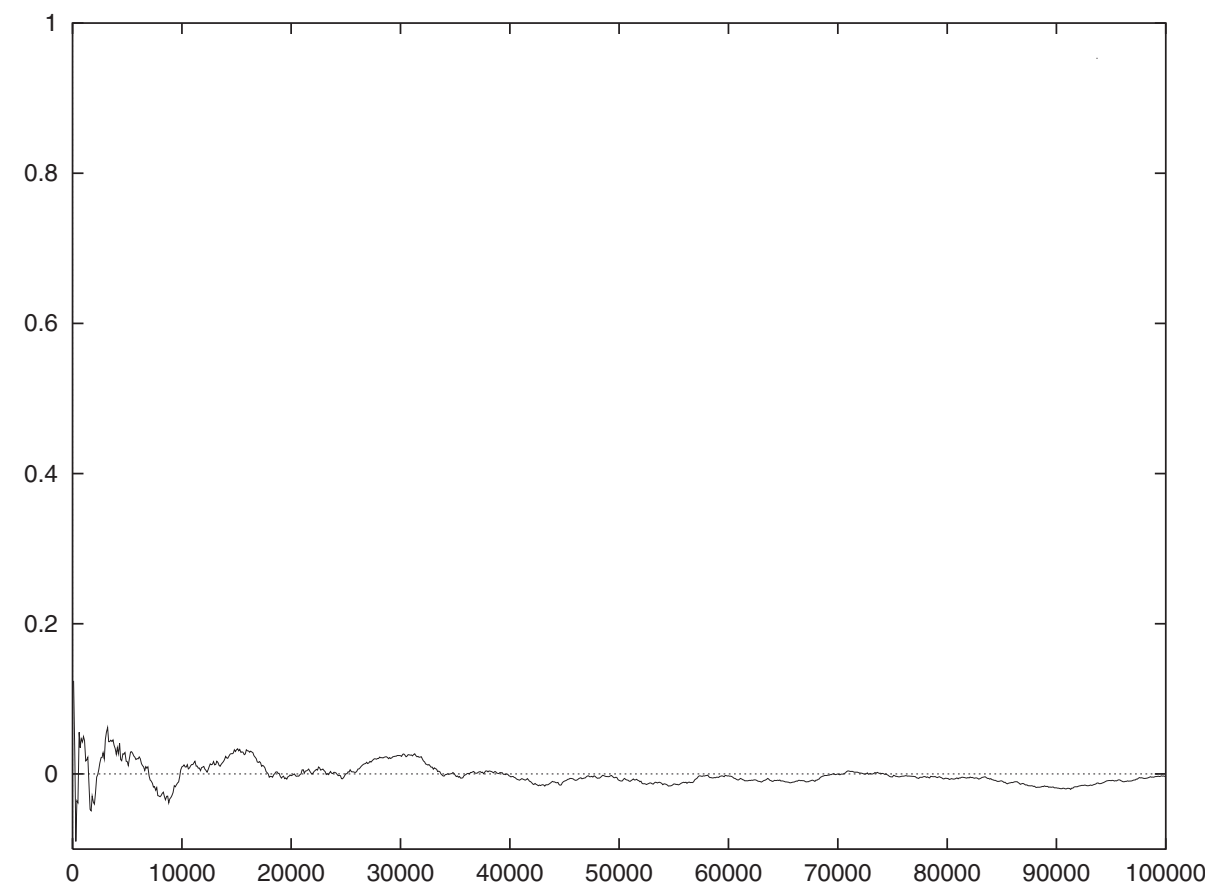

FIGURE 6. The asymptotic behaviour in the case of $3 x^{3}+x^{2}+x$, (imaginary part) $\gamma=\frac{4}{3}$. 


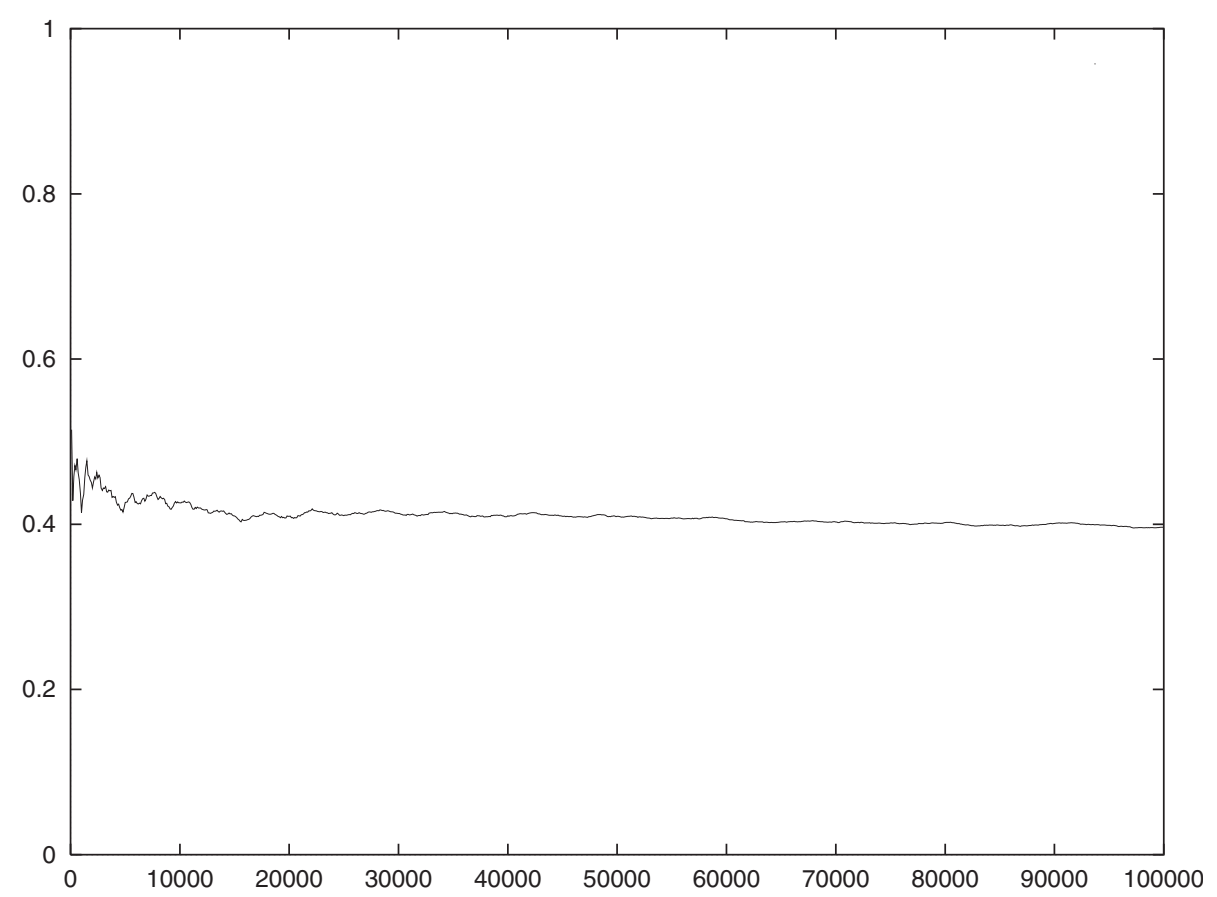

FIGURE 7. The asymptotic behaviour in the case of $x^{4}$ (real part), $\gamma=\frac{3}{2}$.

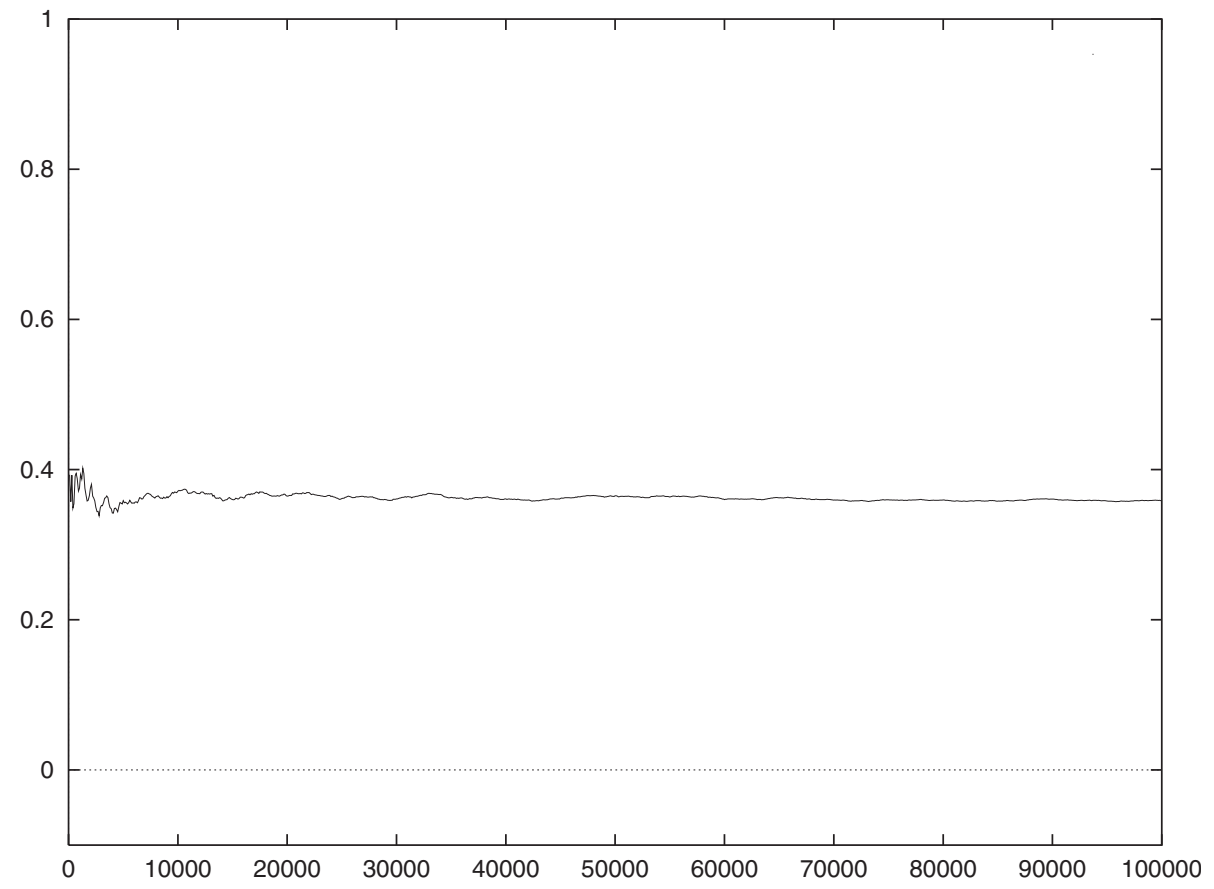

FIGURE 8. The asymptotic behaviour in the case of $x^{4}$ (imaginary part), $\gamma=\frac{4}{3}$. 


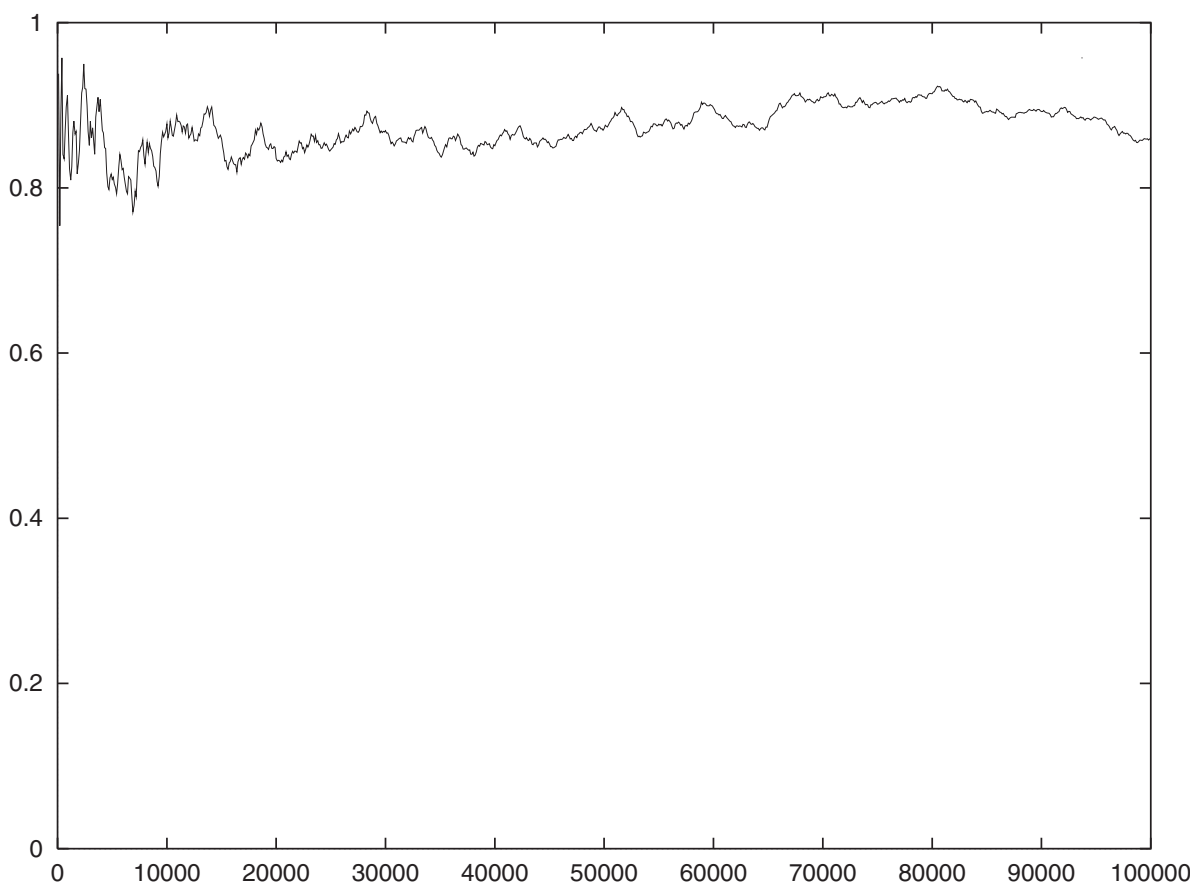

FIGURE 9. The asymptotic behaviour in the case of $x^{4}+x$ (real part), $\gamma=\frac{5}{4}$.

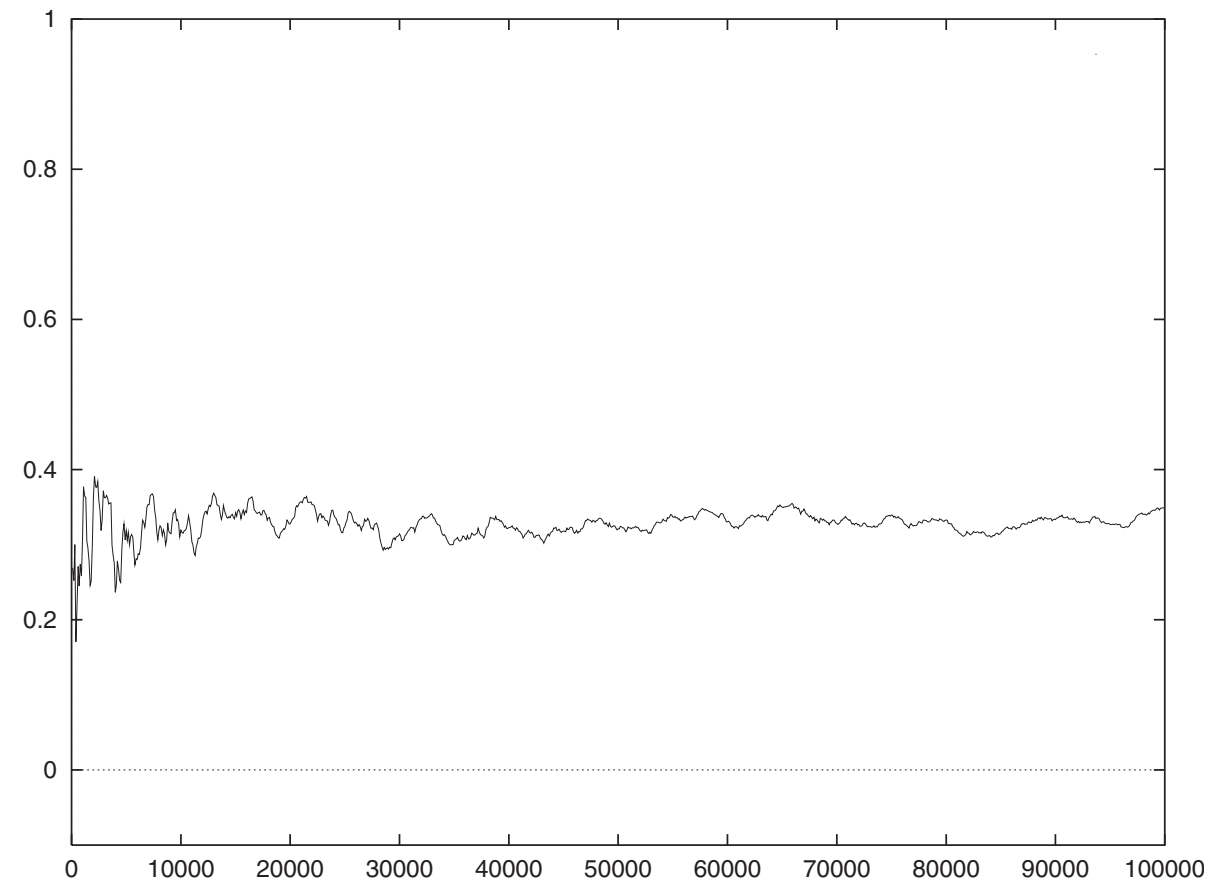

FIGURE 10. The asymptotic behaviour in the case of $x^{4}+x$ (imaginary part), $\gamma=\frac{5}{4}$. 


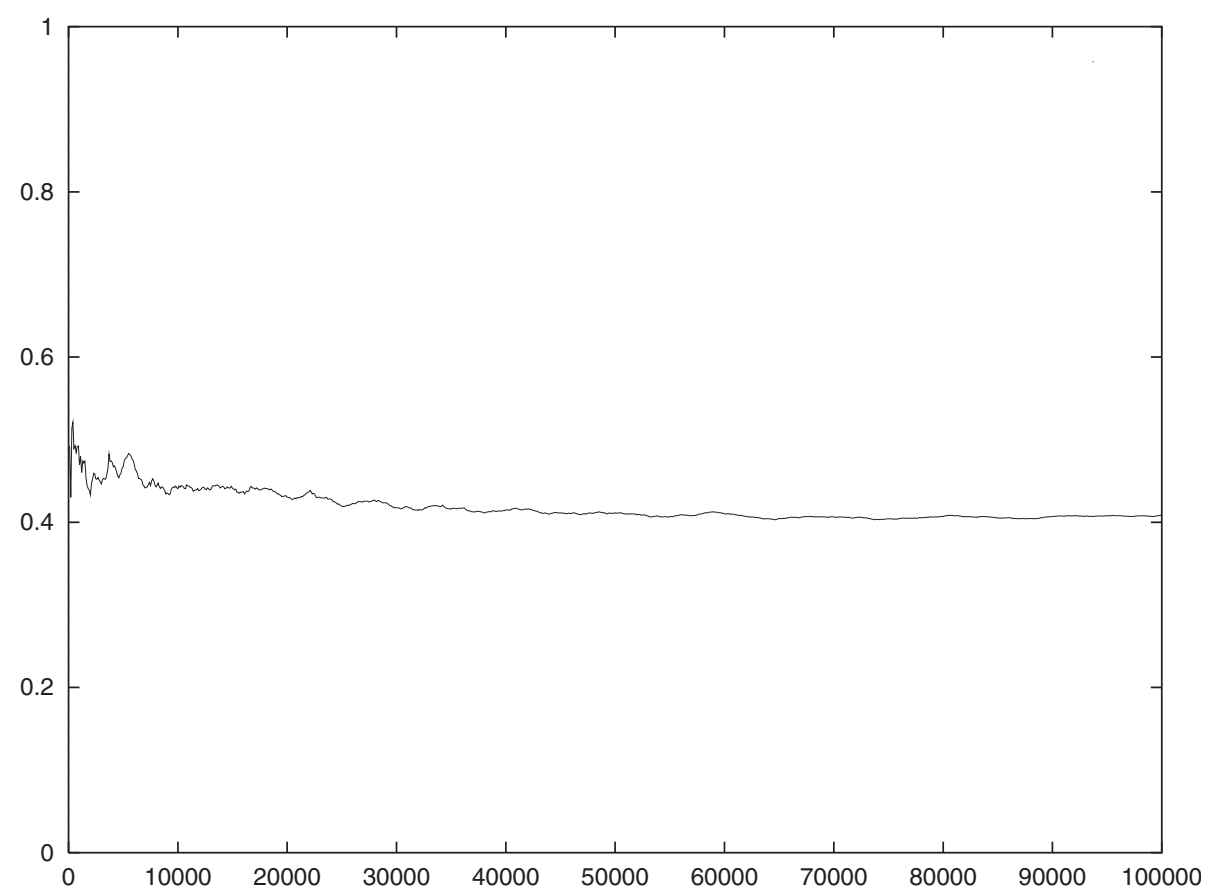

FIGURE 11. The asymptotic behaviour in the case of $x^{4}+x^{2}$ (real part), $\gamma=\frac{3}{2}$.

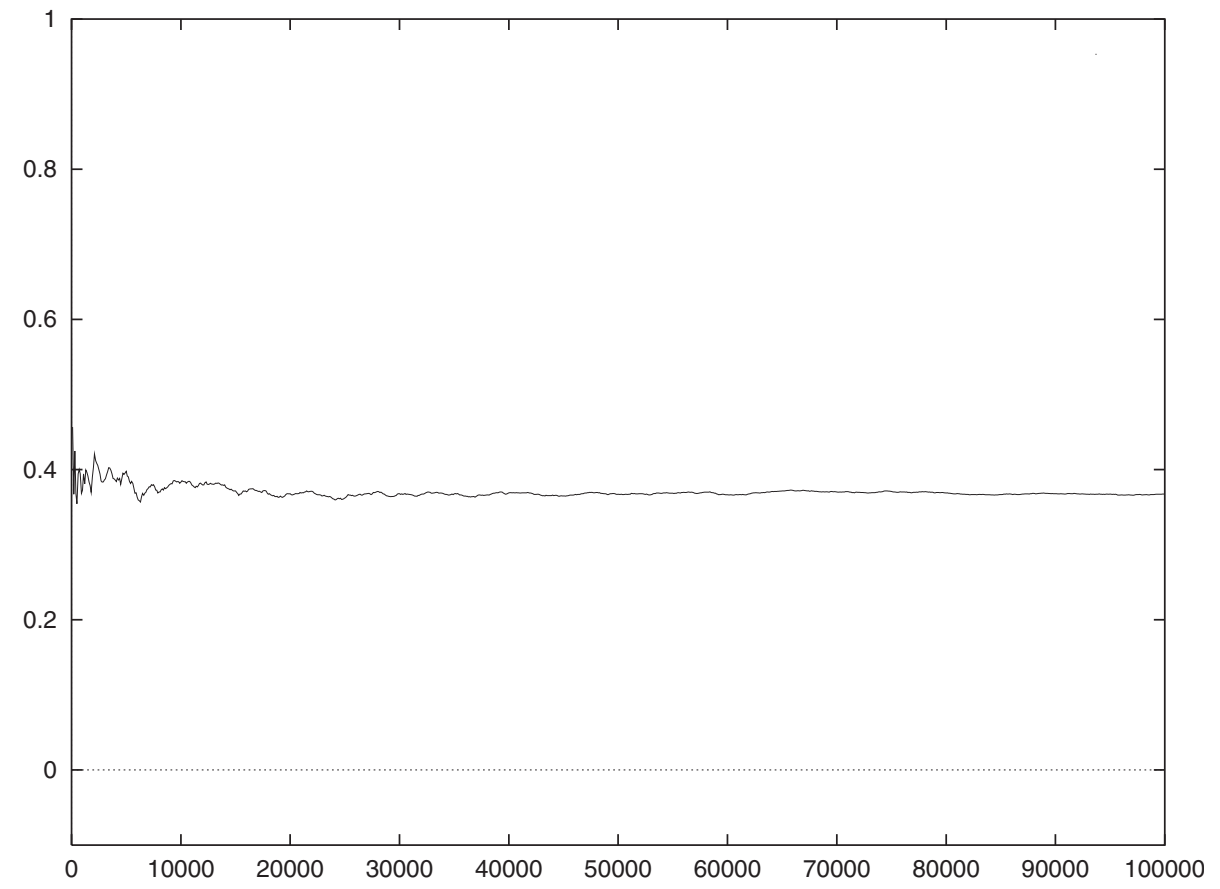

FIGURE 12. The asymptotic behaviour in the case of $x^{4}+x^{2}$ (imaginary part), $\gamma=\frac{3}{2}$. 


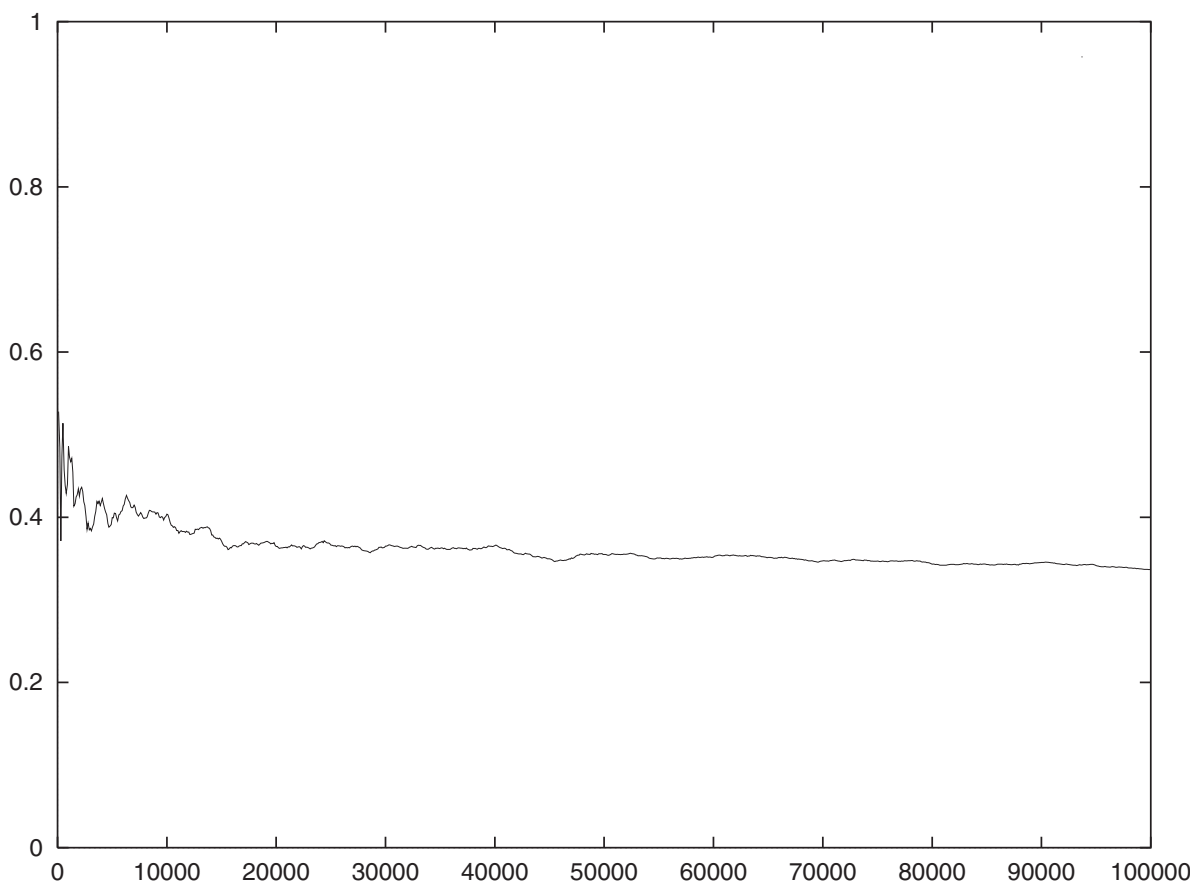

FIGURE 13. The asymptotic behaviour in the case of $2 x^{4}+4 x^{3}+6 x^{2}+4 x+1$ (real part), $\gamma=\frac{3}{2}$.

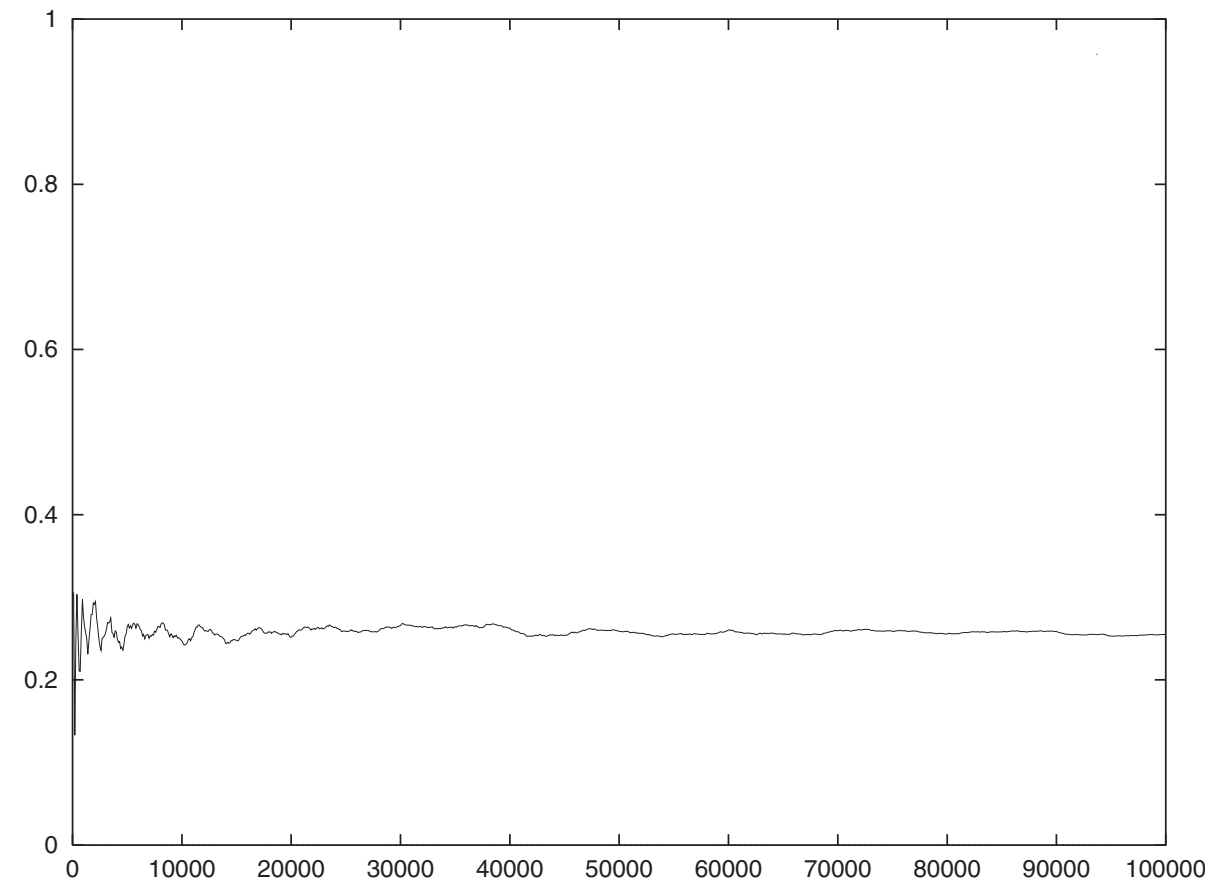

FIGURE 14. The asymptotic behaviour in the case of $2 x^{4}+4 x^{3}+6 x^{2}+4 x+1$ (imaginary part), $\gamma=\frac{3}{2}$. 


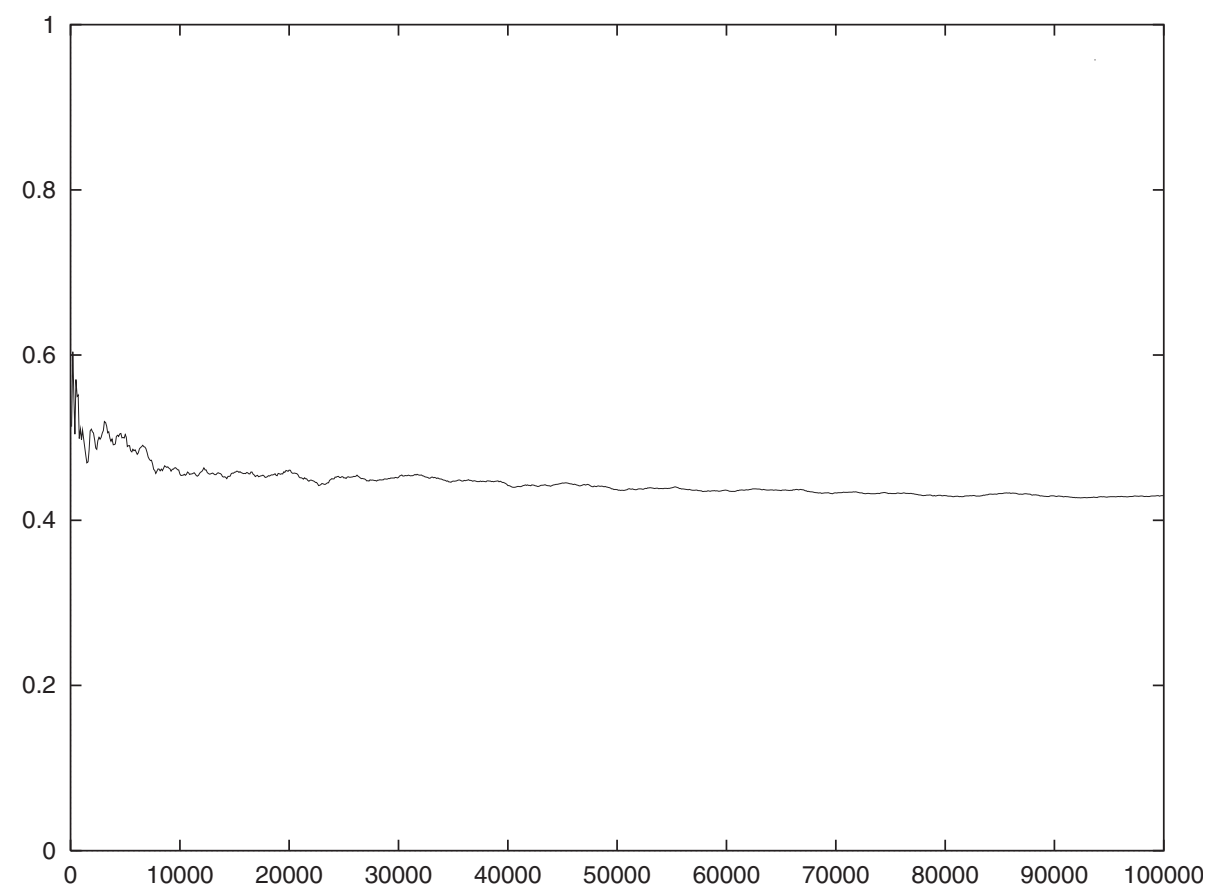

FIGURE 15. The asymptotic behaviour in the case of $x^{4}+2 x^{3}+3 x^{2}+2 x+1$ (real part), $\gamma=\frac{3}{2}$.

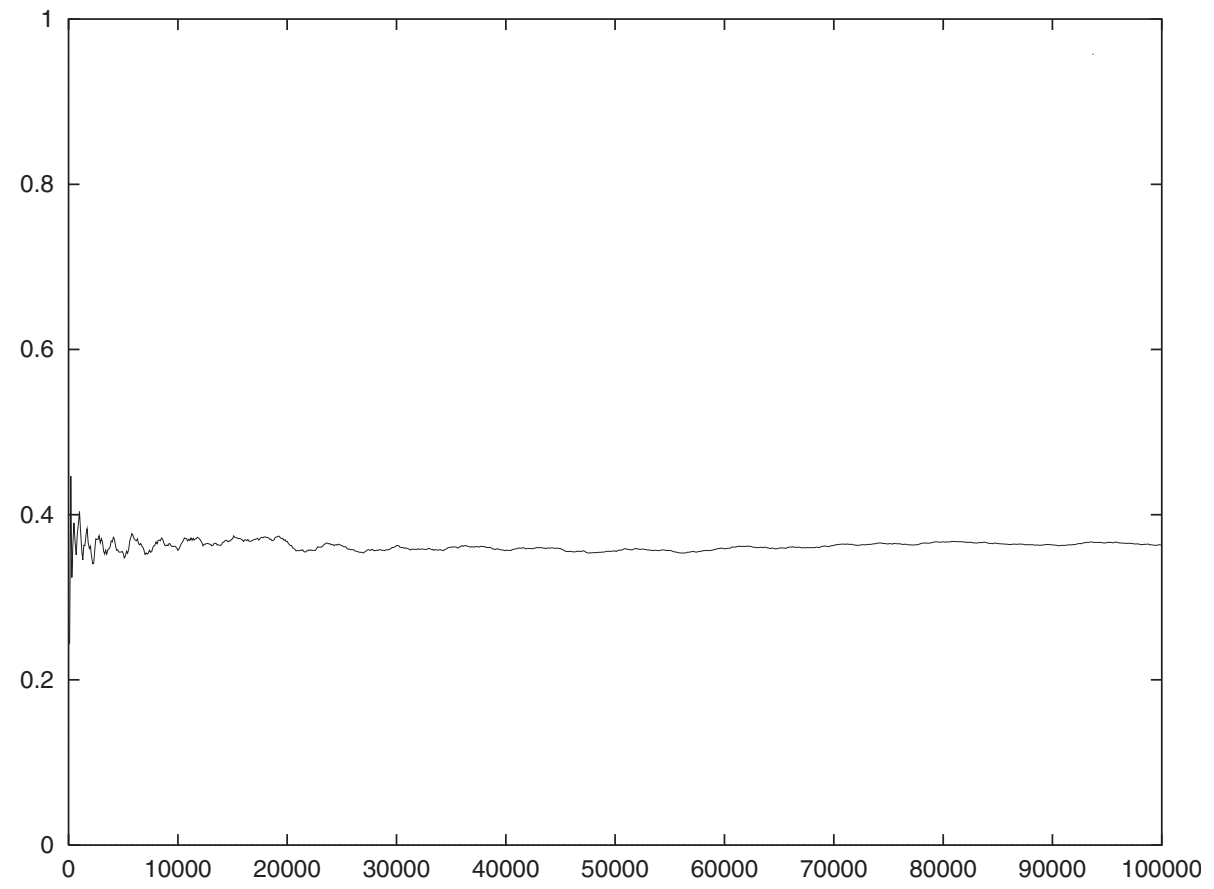

FIGURE 16. The asymptotic behaviour in the case of $x^{4}+2 x^{3}+3 x^{2}+3 x+1$ (imaginary part), $\gamma=\frac{3}{2}$. 


\section{REFERENCES}

[Adolphson 89] A. Adolphson. "On the Distribution of Angles of Kloosterman Sums." J. reine angew. Math. 395 (1989), 214-220.

[Berndt, et al. 98] B. C. Berndt, R. J. Evans, and K. S. Williams. Gauss and Jacobi Sums. New York: WileyInterscience, 1998.

[Copson 65] E. T. Copson. Asymptotic Expansions. Cambridge Tracts 55. Cambridge, UK: Cambridge University Press, 1965.

[Duke et al. 95] W. Duke, J. B. Friedlander, and H. Iwaniec. "Equidistribution of Roots of a Quadratic Congruence to Prime Moduli." Ann. of Math. 141 (1995), 423-441.

[Duke and Iwaniec 93] W. Duke and H. Iwaniec. "A Relation between Cubic Exponential and Kloosterman Sums." Contemp. Math. 143 (1993), 255-258.

[Fouvry and Michel 02] E. Fouvry and P. Michel. "À la recherche de petites sommes exponentielles." Ann. Instit. Fourier 52 (2002), 47-80.

[Fouvry and Michel 03a] E. Fouvry and P. Michel. "Sommes de modules des sommes exponentielles." Pacific J. Math. 209 (2003), 261-288.

[Fouvry and Michel 03b] E. Fouvry and P. Michel. "Sur le changement de signe des sommes de Kloosterman." Preprint.

[Goldfeld and Sarnak 83] D. Goldfeld and P. Sarnak. "Sums of Kloosterman Sums." Inv. Math. 71 (1983), 243-250.

[Heath-Brown 00] D. R. Heath-Brown. "Kummer's Conjecture for Cubic Gauss Sums." Israel J. Math. 120 (2000), 97-124.

[Heath-Brown and Patterson 79] D. R. Heath-Brown and S. J. Patterson. "The Distribution of Kummer Sums at Prime Arguments." J. reine angew. Math. 310 (1979), 111-130.

[Hooley 64] C. Hooley. "On the Distribution of the Roots of Polynomial Congruences." Mathematika 11 (1964), 3949.

[Katz 88] N. Katz. Gauss Sums, Kloosterman Sums and Monodromy Groups. Princeton, NJ: Princeton University Press, 1988.

[Kuznetsov 80] N. V. Kuznetsov. "Petersson Hypothesis for Parabolic Forms of Weight Zero and Linnik Hypothesis. Sums of Kloosterman Sums." Math. Sbornik 111 (1980), $334-383$.

[Linnik 63] Y. V. Linnik. "Additive Problems and Eigenvalues of the Modular Operators." In Proc. Int. Cong. Math. Stockholm, pp. 270-284, Djursholm, Sweden: Institut Mittag-Leffler, 1963.
[Livné and Patterson 02] R. Livné and S. J. Patterson. "The First Moment of Cubic Exponential Sums." Inv. Math. 148 (2002), 79-116.

[Loxton and Smith 82] J. H. Loxton and R. A. Smith. "On Huas's Estimate for Exponential Sums." J. London Math. Soc. 26:2 (1982), 15-20.

[Loxton and Vaughan 85] J. H. Loxton and R. C. Vaughan. "The Estimation of Complete Exponential Sums." Canadian Math. Bull. 28 (1985), 440-454.

[Luo, Rudnick and Sarnak 95] W. Luo, Z. Rudnick, and P. Sarnak. "On Selberg's Eigenvalue Conjecture." GAFA 5 (1995), 387-401.

[Michel 98] P. Michel. "Minorations des sommes d'exponentielles." Duke Math. J. 95 (1998), 227-240.

[Mao 97] Z. Mao. "Airy Sums, Kloosterman Sums and Salié Sums." J. Number Thy. 65 (1997), 316-320.

[Montgomery et al. 95] H. L. Montgomery, R. C. Vaughan, and T. D. Wooley. "Some Remarks on Gauss Sums Associated with $k^{\text {th }}$ Powers." Math. Proc. Cambridge Phil. Soc. 118 (1995), 21-33.

[Murty 85] M. R. Murty. "On the Estimation of the Eigenvalues of Hecke Operators." Rocky Mountain J. Math. 15 (1985) 521-533.

[Patterson 87] S. J. Patterson. "A Heuristic Principle and Applications to Gauss Sums." J. Indian. Math. Soc. 52 (1987), 1-22.

[Patterson 97] S. J. Patterson. "The Asymptotic Distribution of Kloosterman Sums." Acta Arith. 79 (1997), 205-219.

[Patterson 00] S. J. Patterson. "On the Distribution of Certain Hua Sums." Asian J. Math. 4 (2000), 977-986.

[Patterson 02] S. J. Patterson. "On the Distribution of Certain Hua Sums, II." Asian J. Math. 6 (2002), 719-730.

[Selberg 65] A. Selberg. "On the Estimation of the Fourier Coefficients of Modular Forms." Proc. Symp. Pure Math. 8 (1965), 1-15; Collected Papers, Vol. 1, pp. 506-520. Berlin: Springer Verlag, 1989.

[Serre 68] J. P. Serre. Abelian l-Adic Representations and Elliptic Curves, Second edition. Natick, MA: A K Peters, Ltd., 1998.

[Vaughan 81] R. C. Vaughan. The Hardy-Littlewood Method, Cambridge Tracts 80. Cambridge, UK: Cambridge University Press, 1981. 
[Vaughan and Wooley 98 ] R. C. Vaughan and T. D. Wooley. "On the Distribution of Generating Functions." Bull. London Math. Soc. 30 (1998), 113-122.

[Watson 22] G. N. Watson. A Treatise on the Theory of Bessel Functions. Cambridge, UK: Cambridge University Press, 1922.
[Weil 48] A. Weil. "On Some Exponential Sums." Proc. Nat. Acad. Sci. 34 (1948), 204-207; Collected Works, Vol. 1 Berlin: Springer Verlag, 1979.

[Weil 64] A. Weil. "Sur certains groupes d' opérateurs unitaires." Acta Math. 111 (1964), 143-211; Collected Papers, Vol. 3, Berlin: Springer Verlag, 1979.

S. J. Patterson, Mathematisches Institut, Bunsenstr. 3-5, 37073 Göttingen, Germany (sjp@uni-math.gwdg.de)

Received April 1, 2003; accepted in revised form June 25, 2003. 